\title{
Treadmill exercise promotes neurogenesis and myelin repair via upregulating Wnt/ $\beta$-catenin signaling pathways in the juvenile brain following focal cerebral ischemia/reperfusion
}

\author{
JINGYAN CHENG ${ }^{1}$, WEIMIN SHEN ${ }^{1}$, LINGQIN JIN ${ }^{1}$, JUANJUAN PAN ${ }^{1}$, YAN ZHOU ${ }^{1}$, GUOYUAN PAN ${ }^{1}$, \\ QINGFENG XIE ${ }^{1}$, QUAN HU ${ }^{1}$, SHAMIN WU ${ }^{1}$, HONGMEI ZHANG ${ }^{2}$ and XIANG CHEN ${ }^{1}$ \\ ${ }^{1}$ Physical Medicine and Rehabilitation Center, The Second Affiliated Hospital and Yuying Children's Hospital \\ of Wenzhou Medical University, Wenzhou, Zhejiang 325027; ${ }^{2}$ Nursing Department, \\ Hangzhou Children's Hospital, Hangzhou, Zhejiang 310000, P.R. China
}

Received September 12, 2019; Accepted January 20, 2020

DOI: $10.3892 / \mathrm{ijmm} .2020 .4515$

\begin{abstract}
Physical exercise has a neuroprotective effect and is an important treatment after ischemic stroke. Promoting neurogenesis and myelin repair in the penumbra is an important method for the treatment of ischemic stroke. However, the role and potential mechanism of exercise in neurogenesis and myelin repair still needs to be clarified. The goal of the present study was to ascertain the possible effect of treadmill training on the neuroprotective signaling pathway in juvenile rats after ischemic stroke. The model of middle cerebral artery occlusion (MCAO) in juvenile rats was established and then the rats were randomly divided into 9 groups. XAV939 (an inhibitor of the Wnt/ $\beta$-catenin pathway) was used to confirm the effects of the $\mathrm{Wnt} / \beta$-catenin signaling pathway on exercise-mediated neurogenesis and myelin repair. Neurological deficits were detected by modified neurological severity score, the injury of brain tissue and the morphology of neurons was detected by hematoxylin-eosin staining and Nissl staining, and the infarct volume was detected by 2,3,5-triphenyl tetrazolium chloride staining. The changes in myelin were observed by Luxol fast blue staining. The neuron ultrastructure was observed by transmission electron microscopy. Immunofluorescence and western blots analyzed the molecular mechanisms. The results showed that treadmill exercise improved neurogenesis,
\end{abstract}

Correspondence to: Dr Xiang Chen, Physical Medicine and Rehabilitation Center, The Second Affiliated Hospital and Yuying Children's Hospital of Wenzhou Medical University, 109 Xueyuanxi Road, Wenzhou, Zhejiang 325027, P.R. China

E-mail: chenxiangyyfey@163.com

Dr Hongmei Zhang, Nursing Department, Hangzhou Children's Hospital, 195, Wenhui Road, Xiacheng, Hangzhou, Zhejiang 310000, P.R. China

E-mail: 775404664@qq.com

Key words: middle cerebral artery occlusion, treadmill exercise, $\mathrm{Wnt} / \beta$-catenin, neurogenesis, myelin repair, neuroprotection enhanced myelin repair, promoted neurological function recovery and reduced infarct volume. These were the results of the upregulation of Wnt3a and nucleus $\beta$-catenin, brain-derived neurotrophic factor (BDNF) and myelin basic protein (MBP). In addition, XAV939 inhibited treadmill exercise-induced neurogenesis and myelin repair, which was consistent with the downregulation of Wnt3a, nucleus $\beta$-catenin, BDNF and MBP expression, and the deterioration of neurological function. In summary, treadmill exercise promotes neurogenesis and myelin repair by upregulating the $\mathrm{Wnt} / \beta$-catenin signaling pathway, to improve the neurological deficit caused by focal cerebral ischemia/reperfusion.

\section{Introduction}

Stroke is one of the main causes of death and permanent disability worldwide (1). Cerebral artery occlusion can cause acute ischemic stroke. Acute ischemic stroke accounts for $>80 \%$ of all strokes (2). Although some progress has been made in post-stroke treatment, stroke intervention is still insufficient (1). To date, no successful long-term neuroprotective therapy has been identified in clinical trials (3-5). The ischemic core is considered to be an irreparably damaged area. Due to a serious lack of blood flow, numerous nerve cells die within a few minutes after occlusion (6). The ischemic penumbra was first proposed by Astrup et al (7). The penumbra is an area of the brain tissue that is damaged but not yet dead after local ischemia (8). Clinically, the ischemic penumbra is called the low perfusion area around the ischemic core. If the cerebral blood flow is restored in a timely manner, the damaged nerve cells can be saved (9). Neurogenesis (the birth of new neurons) is a process involving the production of functional neurons from precursor cells and occurs throughout the life cycle of the mammalian brain, indicating it is an attractive target for potential intervention $(10,11)$. Most studies have focused on newborn, perinatal and adult rodents, and few have evaluated neurogenesis and myelin repair in adolescents after stroke. Adult neurogenesis is different from developmental neurogenesis (12-14). In the developing brain, immature neurons are extremely sensitive and vulnerable to widespread 
insults and toxic exposures (15). A recent study reported that 3 to 4 -week-old mice have fully developed brains and juvenile mice show mature brain neurons like adult mice, and are not vulnerable to the factors found in neonatal and perinatal brain development (10). Therefore, the juvenile brain is an ideal choice for the study of neurogenesis (10). Elucidation of the signaling molecules and related signaling pathways involved in the protection of nerve cells in the ischemic penumbra after juvenile ischemic stroke is needed.

However, whether in the development of the central nervous system (CNS) or after CNS damage, the Wnt/ $\beta$-catenin signal transduction pathway plays a key role remains to be elucidated (16). It has been found that Wnt3a is an important protein in the Wnt family. It is involved in neurogenesis in the hippocampus and cortex $(17,18)$. Research showed that intranasal administration of Wnt3a can enhance the neuroprotection and regeneration of the Wnt signaling pathway after focal ischemic stroke in mice (19). Previous studies have shown that Wnt signal transduction is the main regulator of hippocampal neurogenesis in adults (20-23). Activating the Wnt pathway in vivo and in vitro was shown to increase neurogenesis, and blocking the Wnt pathway inhibited the proliferation and differentiation of rat neural progenitor cells (NPCs) (20). Moreover, Wnt signaling promotes functional recovery by increasing neurogenesis (24).

Physical exercise can promote neurogenesis, angiogenesis and enhance dendritic modification and synaptic plasticity $(25,26)$. Promoting brain-derived neurotrophic factor (BDNF) expression during development can regulate the cell signal transduction pathway, promote neuronal regeneration and contribute to synaptic plasticity, learning, memory and sensorimotor recovery (27). Treadmill exercise promotes oligodendrocytes and, thus, myelination. In addition, the improvement of ischemia-induced myelin injury by long-term exercise is related to increased BDNF expression (28). Although the authors' previous studies $(25,29,30)$ have focused on the neuroprotective effect of treadmill exercise in adult rats its neuroprotective effect on juvenile rats has not been explored.

In this study, it was shown that treadmill exercise promoted neurogenesis and myelin repair in juvenile rats. The inhibitor of the Wnt signaling pathway was used to elucidate the role of the $\mathrm{Wnt} / \beta$-catenin signaling pathway in treadmill exercise-promoted neurogenesis and myelin repair. It was further clarified that neurogenesis and myelin repair after ischemic stroke are closely related to treadmill exercise and the $\mathrm{Wnt} / \beta$-catenin signaling pathway.

\section{Materials and methods}

Reagents. Anti-Wnt3a (1:1,000; EMD Millipore), anti- $\beta$-catenin (1:5,000; Abcam), anti-BDNF (1:1,000; Abcam), anti-myelin basic protein (MBP; 1:1,000; Abcam), anti-Dcx (1:200; Abcam), anti-NESTIN (1:200; Abcam), anti-GAPDH (1:2,000; Abcam), anti-Lamin B1 (1;1,000; Abcam), XAV939 (Selleck Chemicals), 2,3,5-triphenyl tetrazolium chloride (TTC; Sigma-Aldrich; Merck KGaA), phenylmethylsulfonylfluoride (Beijing Solarbio Science \& Technology Co., Ltd.), Clarity $^{\mathrm{TM}}$ Western ECL Substrate kit (Bio-Rad Laboratories, Inc.), Alexa Fluor 488 AffiniPure Donkey Anti-Mouse IgG and Alexa Fluor 594 AffiniPure Goat Anti-Rabbit IgG (1:200; Yesen Bio), 4,6-diamidino-2-phenylindole (DAPI; Beyotime Institute of Biotechnology).

Animals. The Shanghai Experimental Animal Center provided 171 juvenile Sprague-Dawley male rats (weight, 80-90 g; Sprague Dawley rats were weaned for 21 days and matured for 6 to 7 weeks). The Animal Research Committee of Wenzhou Medical University approved the present experimental project and all the experimental followed the guidelines of the National Institutes of Health on the Care and Use of Animals (ethical no. wydw2019-0766). The animals were placed in a suitable environment (4 rats per cage, relative humidity $55 \pm 5 \%, 22^{\circ} \mathrm{C}$, 12-h light/dark cycle) and had free access to food and water. The rats were randomly divided into 9 groups: Sham operation group ( $\mathrm{n}=19 ; \mathrm{S}$ group); model group 7 and 14 days, respectively (M7: $n=19$ and M14: $n=19$ ); treadmill training models (EM7: $\mathrm{n}=19$ and EM14: $\mathrm{n=19}$ ); inhibitor treatment models (IM7: $\mathrm{n}=19$ and IM14: $\mathrm{n=19}$ ); and inhibitor treatment and treadmill training models (IEM7: $n=19$ and IEM14: $n=19$ ). The present research was conducted following these criteria (31): i) Randomization, ii) allocation concealment and iii) blinded assessment of outcome.

Middle cerebral artery occlusion (MCAO) model offocal brain ischemia. Briefly, rats were anesthetized with $3 \%$ isoflurane vaporized in $30 \% \mathrm{O}_{2} / 70 \% \mathrm{~N}_{2}$ until they were unresponsive to the tail pinch test and then fitted with a nose cone blowing $1.5 \%$ isoflurane for anesthesia maintenance (Shenzhen RWD Life Science Co., Ltd.) during surgery. After the rats were anesthetized, they were placed in the supine position on the sterilized operating table and an incision was made in the right side of the neck. The common carotid artery and external carotid artery (ECA) was separated from the peripheral connective tissue without injury to peripheral muscles and nerves. A small gap was opened in the stump of the ECA and then a small silicon-coated surgical nylon monofilament (0.24 $\pm 0.02 \mathrm{~mm} / 1,2,400$; Guangzhou Jialing Biotechnology Co., Ltd.) was inserted into the lumen of the internal carotid artery (ICA), and the operation was stopped $\sim 17 \mathrm{~mm}$ from the far end of the ICA bifurcation to block the middle cerebral artery (MCA). The rats during and after the operation were preserved under a $37^{\circ} \mathrm{C}$ heating pad. After $1.5 \mathrm{~h}$ of occlusion, the filament in the rat ICA was removed under a light microscope (Olympus Corporation) and the blood flow was restored, and the reperfusion was allowed for 7 or 14 days. The rats in the sham operation group were treated with the same surgical method as the experimental group except for the insertion of the surgical nylon monofilament. A total of 59 rats were excluded from the present study, including 14 rats with low Zea Longa score ( 0 or 4 ) (32) and 45 rats that died. Among the 45 rats that died, 14 rats died of brain edema, 9 died of subarachnoid hemorrhage, 13 died of massive haemorrhage and 9 died of long operation time. In addition, all juvenile rats were anesthetized by intraperitoneal injection of pentobarbital sodium $(65 \mathrm{mg} / \mathrm{kg})$ before sacrifice.

Treadmill exercise and inhibitor. In this study, a small animal electric treadmill was used (XR-PT-10A; Shanghai XinRuan Information Technology Co., Ltd.). Before MCAO, the animals 
received three days of adaptive treadmill training. The EM group and IEM group underwent treadmill training at 0 slope, $8 \mathrm{~m} / \mathrm{min}, 30 \mathrm{~min} / \mathrm{d}$ and $5 \mathrm{~d} /$ for 7 days or 14 days, respectively.

XAV939, a small molecule inhibitor of Wnt/ $\beta$-catenin signaling (40 mg/kg; intraperitoneal injection) was used. In short, immediately after MCAO, XAV-939 was injected into the IM7 group and the IEM7 group, and then injected every $24 \mathrm{~h}$ for 7 days. Also, in IM14 group and IEM14 group, immediately after MCAO, XAV-939 was injected into IM14 group and IEM14 group, and then injected every $24 \mathrm{~h}$ for 14 days. the dose of XAV939 was determined based on dose-response studies and published reports $(33,34)$. In addition, rats of the other groups ( $\mathrm{S}, \mathrm{M}$ and $\mathrm{EM}$ ) were simultaneously injected with the same amount of physiological saline.

Evaluation of neurologic deficit scores. A total of two independent examiners performed the modified nerve severity score (mNSS) test (35) at 1, 7 and 14 days after MCAO. The researchers were blinded to the treatment group (Table I).

Infarct volume assessment. For determination of the volume of cerebral infarction, TTC staining was performed on consecutive sections of bregma $+4.0-6.0 \mathrm{~mm}$. the brain was cut with a blade into five consecutive coronal sections separated by $2.0 \mathrm{~mm}$. The obtained brain tissue was frozen at $-20^{\circ} \mathrm{C}$ for $10 \mathrm{~min}$. Then, all brain slices were immediately immersed in $2 \%$ TTC solutions, at $37^{\circ} \mathrm{C}$ for $30 \mathrm{~min}$ and fixed at $4^{\circ} \mathrm{C}$ for $24 \mathrm{~h}$ in $4 \%$ paraformaldehyde buffer. In 5 sections, the total cerebral infarction volume was equal to the sum of the infarction area. The correction formula for calculating the infarction volume with minimization of the error caused by brain edema is as follows (36): Infarct percentage $=[($ contralateral hemisphere region-non-infarcted region in the ipsilateral hemisphere)/volume of the contralateral hemisphere] $* 100 \%$. Then, the analysis was carried out using ImageJ software (ImageJ bundled with 64-bit Java 1.8.0_112; National Institutes of Health).

Bromodeoxyuridine injection and tissue preparation. Bromodeoxyuridine (BrdU; $50 \mathrm{mg} / \mathrm{kg} /$ day) was injected intraperitoneally into rats every day after MCAO for 7 or 14 days to label newly formed cells. The proliferation of neural stem cells (NSCs) and the differentiation of NSCs were observed by double immunofluorescence.

At 7 and 14 days after MCAO, 3 rats in each group were sacrificed. Under deep anesthesia with pentobarbital sodium (65 $\mathrm{mg} / \mathrm{kg}$; intraperitoneally), the rats were perfused with $0.9 \%$ sodium chloride $\left(4^{\circ} \mathrm{C}\right)$ and then perfused with $4 \%$ paraformaldehyde (Sigma-Aldrich; Merck KGaA) in $0.1 \mathrm{~mol} / 1$ phosphate buffer ( $\mathrm{pH}$ 7.4). The whole brain was collected, fixed at $4^{\circ} \mathrm{C}$ for $24 \mathrm{~h}$, dehydrated and finally embedded in paraffin wax. Then, coronal sections of $5 \mu \mathrm{m}$ were sliced by a microtome (Kedee Instrumental Equipment Co. Ltd.) for hematoxylin-eosin $(\mathrm{H} \& \mathrm{E})$ or Nissl staining. The rat samples used for immunofluorescence labeling were removed and stored in the same fixation solution at $4^{\circ} \mathrm{C}$ for $24 \mathrm{~h}$ and soaked overnight in $0.1 \mathrm{M}$ phosphate buffer (20 and 30\% sucrose) at $4^{\circ} \mathrm{C}$. The brain tissue was embedded and frozen at $-80^{\circ} \mathrm{C}$ in the optimal cutting temperature complex. Finally, the coronal section of the ischemic penumbra was cut with a cryostat (CM1900; Leica Microsystems GmbH) (5 $\mu \mathrm{m}$ thick).
Table I. Modified neurological severity score points.

Motor tests

Raising rat by tail

Flexion of forelimb

1

Flexion of hindlimb

Head moved 10 to vertical axis within $30 \mathrm{sec}$

Placing rat on floor (normal=0; maximum=3)

Normal walk

Inability to walk straight

Circling toward paretic side

Falls down to paretic side

Sensory tests

Placing test (visual and tactile test)

Proprioceptive test (deep sensation, pushing paw

against table edge to stimulate limb muscles)

Beam balance tests (normal $=0$; maximum=6)

Balances with steady posture

Grasps side of beam

Hugs beam and 1 limb falls down from beam

Hugs beam and 2 limbs fall down from beam, or spins on beam ( $>60 \mathrm{sec}$ )

Attempts to balance on beam but falls off ( $>40 \mathrm{sec})$

Attempts to balance on beam but falls off ( $>20 \mathrm{sec}$ )

Falls off; no attempt to balance or

hang on to beam $(<20 \mathrm{sec})$

Reflex absence and abnormal movements

Pinna reflex (head shake when auditory

meatus is touched)

Corneal reflex (eye blink when cornea is

lightly touched with cotton)

Startle reflex (motor response to a brief noise

from snapping a clipboard paper)

Seizures, myoclonus, myodystony

Maximum points

One point is awarded for inability to perform the tasks or for lack of a tested reflex: 13-18, severe injury; 7-12, moderate injury; 1-6, mild injury.

$H \& E$ staining. At least 3 slices were taken from each rat, dewaxed in xylene and then dehydrated in alcohol. Finally, $\mathrm{H} \& \mathrm{E}$ staining was performed at room temperature for $2 \mathrm{~h}$, and observed under a light microscope (Olympus Corporation). Pathological changes in brain tissue were observed, images were captured and recorded.

Toluidine blue (Nissl) staining. The slices were washed 3 times. The slices were dyed at $37^{\circ} \mathrm{C}$ with $1 \%$ toluene blue solution for $30 \mathrm{~min}$. The tissue was decolorized and dehydrated with ethanol and sealed with neutral resin. Under light microscopy (Olympus Corporation), the Nissl bodies of neurons were blue and purple.

Luxol fast blue (LFB) staining. The brain tissue was embedded in paraffin and then, the $5 \mu \mathrm{m}$ thick coronal slices 
A

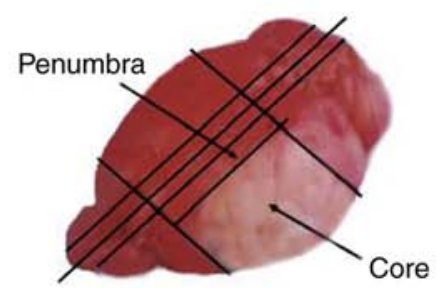

B

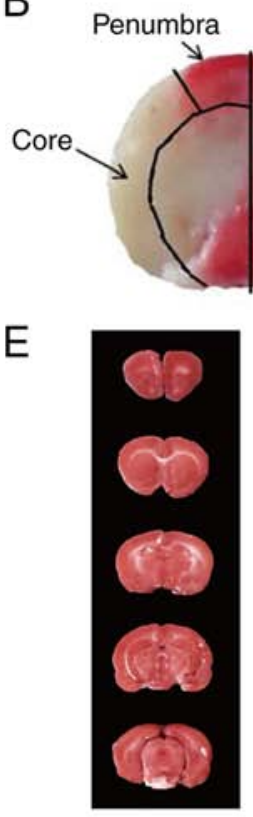

S

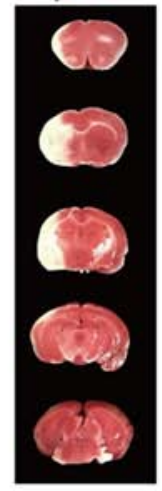

M7

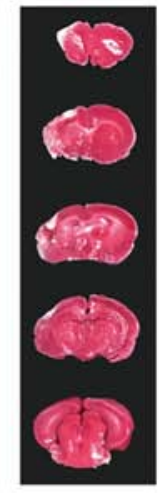

EM7

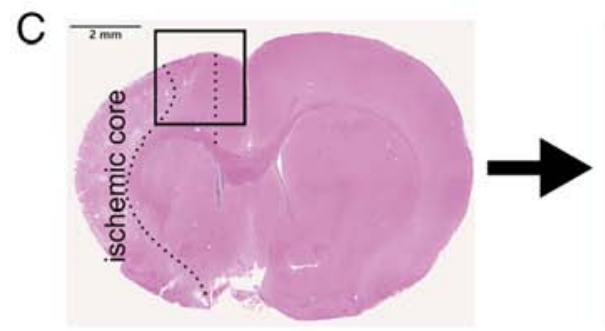

D
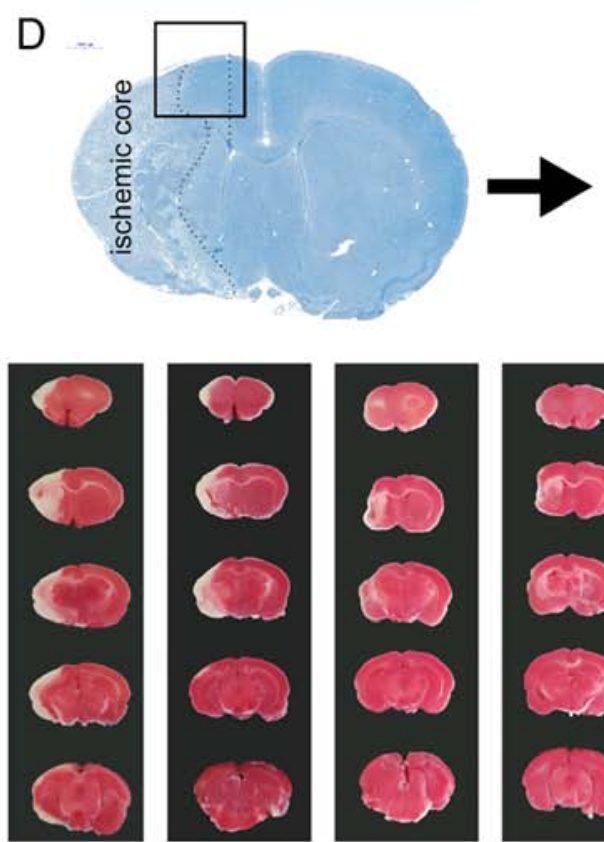

IM7

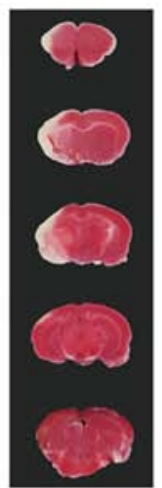

IEM7

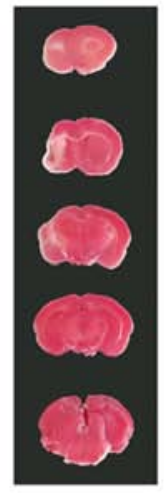

M14

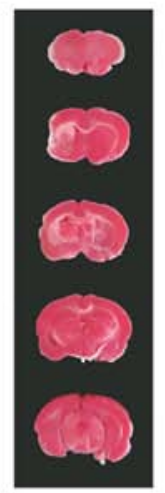

EM14
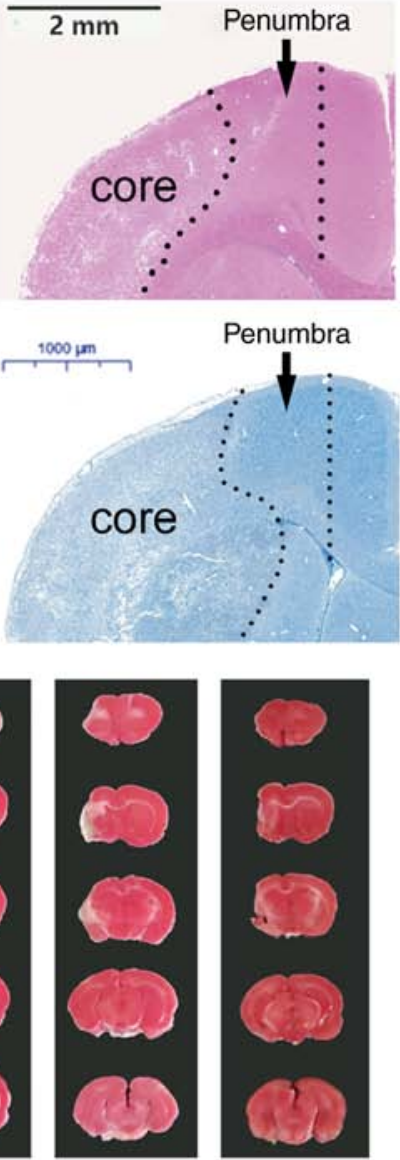

IM14

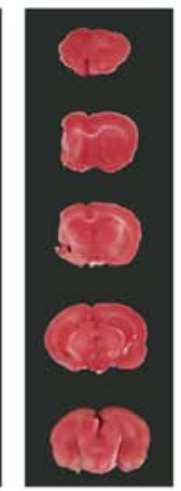

IEM14
F

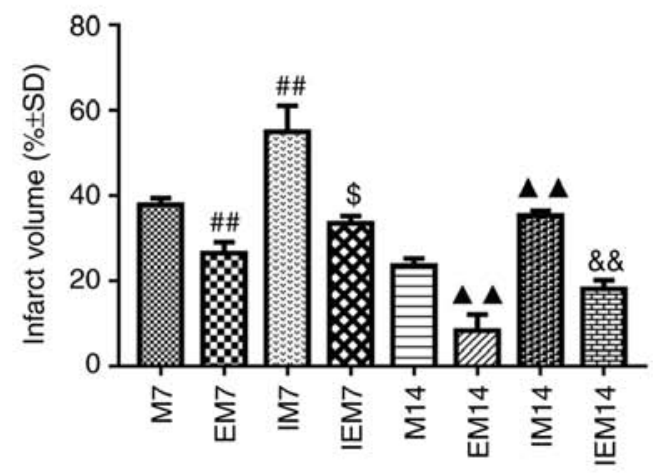

G

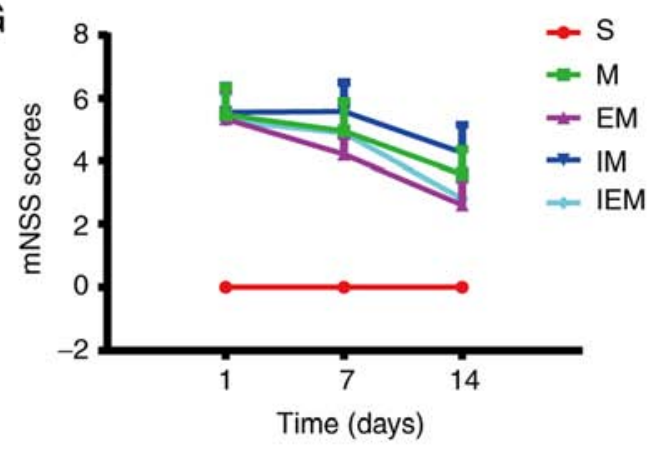

Figure 1. Treadmill training attenuates neurological deficits and decreases infarct size, while the inhibitor increased infarct injury. (A and B) Site of ischemic core and ischemic penumbra area for the same rat. (A) Overall look for the site of ischemic core and ischemic penumbra area. (B) Local image on site of ischemic core and ischemic penumbra area; picture from the previous paper of the current team (24). (C) Hematoxylin \& eosin staining. Scale bars=2 mm. (D) Nissl staining. Scale bars $=1,000 \mu \mathrm{m}$. (C) and (D) are shown the location of ischemic penumbra. (E) Showed infarct volumes were assessed by 2,3,5-triphenyl tetrazolium chloride. (F) Percentage of infarct volume in each group. Data are presented as the mean \pm standard deviation ( $=5$ ). ${ }^{\#} \mathrm{P}<0.001 \mathrm{vs}$. the $\mathrm{M} 7$ group; ${ }^{\$} \mathrm{P}<0.05$ vs. the EM7 group; ${ }^{\boldsymbol{\Lambda}} \mathrm{P}<0.01$ vs. the M14 group; ${ }^{\&} \mathrm{P}<0.01$ vs. the EM14 group. (G) Neurological scores in different groups, in which EM7 vs. M7, P<0.01; EM14 vs. M14, P<0.01; IM7 vs. M7, P<0.05; IM14 vs. M14, P<0.01; IEM7 vs. EM7, P<0.01; IEM14 vs. EM14, P>0.05. M group, model group; EM group, treadmill training model group; mNSS, modified nerve severity score.

were stained with $0.1 \%$ LFB (cat. no. S3382; Sigma-Aldrich; Merck $\mathrm{KGaA}$ ) at $60^{\circ} \mathrm{C}$ for $2 \mathrm{~h}$. The slices were soaked in $0.05 \%$ lithium carbonate solution to distinguish white matter from gray matter. Finally, the slices were placed in distilled water, re-dyed at room temperature in cresol purple solution for 30-40 sec and then washed with distilled water.

Immunofluorescence. The slices were dried, then restored to room temperature and soaked in $0.01 \mathrm{M}$ phosphate buffer
(PBS, pH 7.6) 3 times for 5 min. The sections were blocked for $1 \mathrm{~h}\left(20-22^{\circ} \mathrm{C}\right)$ with $10 \%$ goat serum (cat. no. C0265; Beyotime Institute of Biotechnology) containing 0.3\% Triton X-100. Dcx/BrdU and Nestin/BrdU double immunofluorescence staining was performed. Samples were incubated at $37^{\circ} \mathrm{C}$ with 1N HCL for $30 \mathrm{~min}$ before blocking and then denatured twice for $10 \mathrm{~min}$ with borate buffer $(\mathrm{pH} 8.4)$. Then mixtures of mouse anti-BrdU antibody (dilution, 1:1,000; cat. no. MAB4072; EMD Millipore) and rabbit anti-Dcx antibody 
(dilution, 1:200; cat. no. ab207175; Abcam), mixtures of mouse anti-BrdU antibody and rabbit anti-Nestin antibody (dilution, 1:200; cat. no. ab105389; Abcam), and rabbit anti-BDNF antibody (dilution, 1:100; cat. no. ab108319; Abcam), used as antibodies for Dcx/BrdU, Nestin/BrdU and BDNF, respectively, were separately added for incubation overnight at $4^{\circ} \mathrm{C}$. Next, the sections were restored to room temperature. Then, the slices were soaked in 0.01 M PBS 3 times for $5 \mathrm{~min}$ and performed with the corresponding Alexa Fluor 488 AffiniPure Donkey Anti-Mouse IgG (dilution, 1:200; cat. no. 34106ES60; Shanghai Yeasen Biotechnology Co., Ltd.) and Alexa Fluor 594 AffiniPure Goat Anti-Rabbit IgG (dilution, 1:200; cat. no. 33112ES60; Shanghai Yeasen Biotechnology Co., Ltd.) secondary antibodies for $1 \mathrm{~h}$ at room temperature. Nuclei were counterstained at room temperature for $10 \mathrm{~min}$ with DAPI. The negative control sections were incubated with 0.01 M PBS instead of primary antibody and no positive fluorescence signal was found. According to the present team's previous explorations and classical anatomical methods, the area of observation of the ischemic penumbra is as shown in Fig. 1C and D (37). Brain sections were covered and the ischemic penumbra was observed and analyzed by fluorescence microscopy (BX51; Olympus Corporation) in all sections. Counting the positive cells of 5 non-overlapping fields (x400-fold) on one slice using Image-Pro Plus 5.0 analysis software (Media Cybernetics, Inc.).

Western blot analysis. As in the authors' previous publication (26), coronal sections were performed at 4 and $9 \mathrm{~mm}$ from the prefrontal lobe, and then the left and right brains were separated. Finally, sagittal sections were performed on the $1.0-1.5 \mathrm{~mm}$ from the midline of the brain. a transverse diagonal cut was then performed at '10 o'clock' to separate the core from the penumbra (38) (Fig. 1A and B). According to the protocol of rat brain tissue extraction kit (Beyotime Institute of Biotechnology), total protein and nuclear protein were extracted respectively. The protein concentration was detected by a BCA protein detection kit (Beyotime Institute of Biotechnology). The same amount of protein $(50 \mu \mathrm{g})$ was transferred to a polyvinylidene difluoride (EMD Millipore) membrane after $10 \%$ SDS-PAGE analysis. The membranes were blocked with 5\% skim milk at room temperature for $2 \mathrm{~h}$. Wnt3a, $\beta$-catenin, BDNF, MBP, GAPDH and Lamin B1 were incubated on a $4^{\circ} \mathrm{C}$ shaking table overnight. TBS with $0.05 \%$ Tween-20 was washed three times and then, the membranes were soaked in horseradish peroxidase-conjugated secondary antibody (HRP AffiniPure Goat Anti-Rabbit IgG (H+L); dilution, 1:10,000; cat. no. E030120; EarthOx, LLC) at room temperature for $1.5 \mathrm{~h}$. The protein imaging was detected by a Clarity ${ }^{\mathrm{TM}}$ Western ECL Substrate kit (cat. no. 1705060; Bio-Rad Laboratories, Inc.). ImageJ software (National Institute of Health) was used for quantitative analysis.

Transmission electron microscopy (TEM). The rats were sacrificed at 7 and 14 days after MCAO, and ultrastructural changes of neurons in the ischemic penumbra cortex was observed by TEM. The specimens were fixed for at least $2 \mathrm{~h}$ with $2.5 \%$ (w/v) glutaraldehyde overnight, washed three times for $10 \mathrm{~min}$ in PBS and then soaked with $2 \%(\mathrm{v} / \mathrm{v})$ osmium tetroxide at $37^{\circ} \mathrm{C}$ for $1 \mathrm{~h}$. The specimens were taken
Table II. Modified neurological severity scores post middle cerebral artery occlusion.

\begin{tabular}{lcccc}
\hline Group & $\mathrm{N}$ & 1 day & 7 days & 14 days \\
\hline $\mathrm{S}$ & 19 & 0 & 0 & 0 \\
M & 38 & $5.47 \pm 0.89^{\mathrm{a}}$ & $4.95 \pm 0.96^{\mathrm{a}}$ & $3.58 \pm 0.79^{\mathrm{a}}$ \\
EM & 38 & $5.34 \pm 0.94$ & $4.21 \pm 0.58^{\mathrm{b}}$ & $2.61 \pm 0.82^{\mathrm{b}}$ \\
IM & 38 & $5.55 \pm 0.83$ & $5.58 \pm 0.92^{\mathrm{b}}$ & $4.26 \pm 0.89^{\mathrm{b}}$ \\
IEM & 38 & $5.29 \pm 0.90$ & $4.89 \pm 0.83^{\mathrm{c}}$ & $2.81 \pm 0.90$ \\
\hline
\end{tabular}

Data were presented as mean \pm standard deviation, ANOVA. ${ }^{a} \mathrm{P}<0.05$ vs. the same period of $\mathrm{S}$. ${ }^{\mathrm{b}} \mathrm{P}<0.05$ vs. the same period of $\mathrm{M}$. ${ }^{\mathrm{c}} \mathrm{P}<0.05$ vs. the same period of EM. S group, sham operation group; M group, model group; EM group, treadmill training model group; IM group, inhibitor treatment model group; IEM group, Inhibitor treatment and treadmill training model group.

Table III. Results of infarct volume in each group after middle cerebral artery occlusion (\%).

\begin{tabular}{lccc}
\hline Group & $\mathrm{N}$ & 7 days & 14 days \\
\hline S & 3 & 0 & 0 \\
M & 5 & $37.92 \pm 1.50^{\mathrm{a}}$ & $23.37 \pm 1.75^{\mathrm{a}}$ \\
EM & 5 & $26.28 \pm 2.68^{\mathrm{b}}$ & $8.19 \pm 3.86^{\mathrm{b}}$ \\
IM & 5 & $54.89 \pm 6.21^{\mathrm{b}}$ & $35.25 \pm 1.20^{\mathrm{b}}$ \\
IEM & 5 & $33.41 \pm 1.77^{\mathrm{c}}$ & $18.05 \pm 2.12^{\mathrm{c}}$
\end{tabular}

Data were presented as mean \pm standard deviation, ANONE. ${ }^{a} \mathrm{P}<0.05$ vs. the same period of $\mathrm{S}$. ${ }^{\mathrm{b}} \mathrm{P}<0.05$ vs. the same period of $\mathrm{M}$. ${ }^{\mathrm{c}} \mathrm{P}<0.05$ vs. the same period of EM. S group, sham operation group; M group, model group; EM group, treadmill training model group; IM group, inhibitor treatment model group; IEM group, Inhibitor treatment and treadmill training model group.

out and rinsed twice, then stained at room temperature for $2 \mathrm{~h}$ with $1 \%$ uranyl acetate and dehydrated with acetone. After dehydration, the tissue was embedded in the mixture of acetone and entrapped liquid (1:1) for incubation at $37^{\circ} \mathrm{C}$ for $2 \mathrm{~h}$, followed by maintenance in mixture of acetone and entrapped liquid (1:4) for incubation at $37^{\circ} \mathrm{C}$ overnight. After that, the tissue was soaked in entrapped liquid for $2 \mathrm{~h}$ at $45^{\circ} \mathrm{C}$, followed by incubation at $45^{\circ} \mathrm{C}$ for $3 \mathrm{~h}$ and $65^{\circ} \mathrm{C}$ for $48 \mathrm{~h}$ to obtain the coronal sections. Semi-thin sections and toluidine blue staining were used for localization observation. Finally, at least three ultra-thin sections in each sample were observed by TEM (JEM-1200EX; JEOL, Ltd.).

Statistical analysis. All analyses were performed using GraphPad Prism 4.0 (GraphPad Software, Inc.) or IBM SPSS 25.0 statistical software (IBM Corp.). Multiple comparisons were carried out by one-way analysis of variance (ANOVA) with Tukey (when equal variances assumed) or Dunnett's T3 (when equal variances not assumed) post-hoc analysis. $\mathrm{P}<0.05$ was considered to indicate a statistically significant difference. The data are reported as the mean \pm standard deviation. 
A

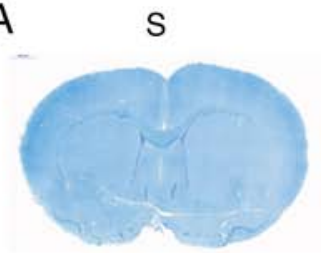

M7

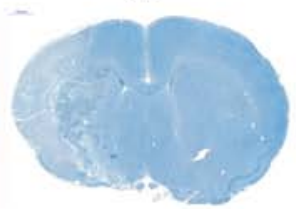

EM7

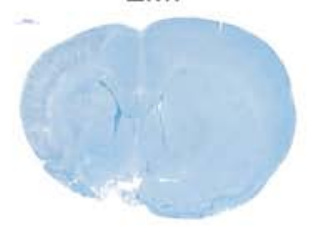

IM7

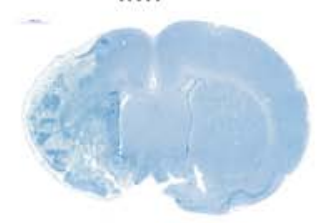

IEM7

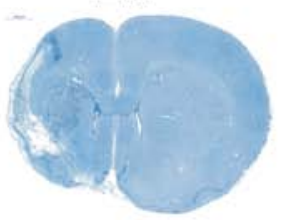

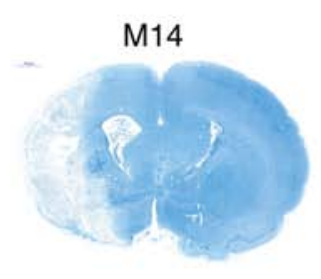

B

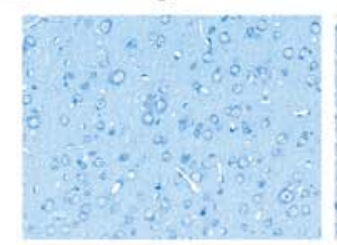

C

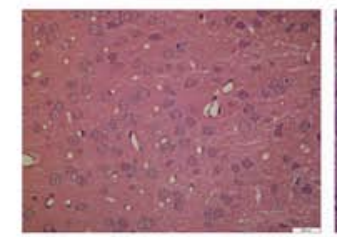

EM14

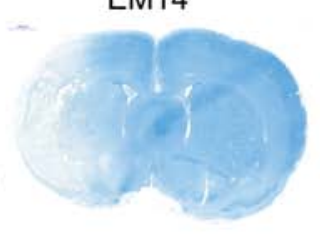

M

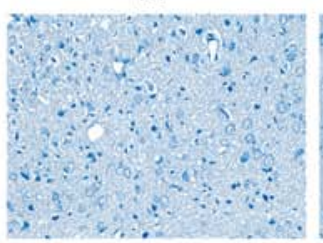

M

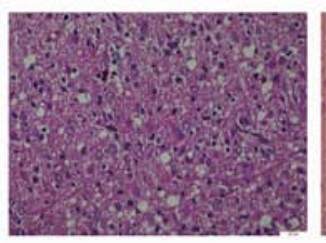

IM14

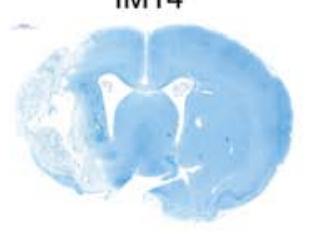

EM

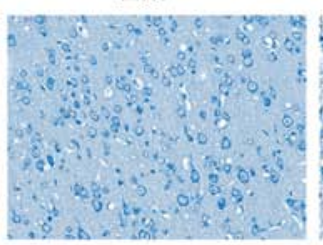

EM

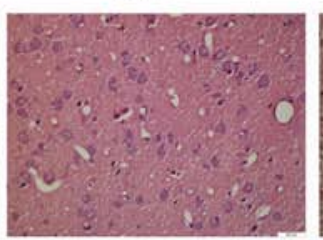

IM

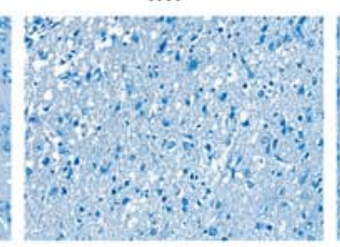

IM

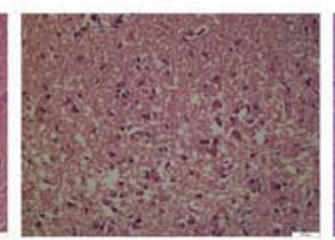

IEM14

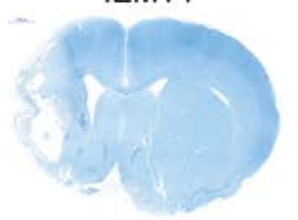

IEM

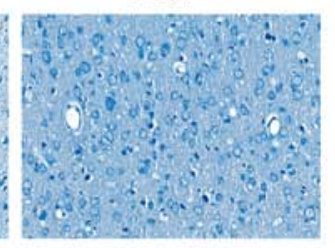

IEM

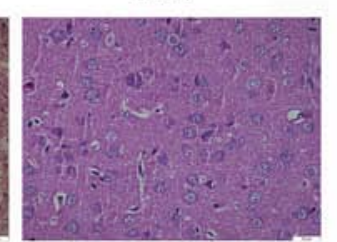

Figure 2. Treadmill training decreases the damage of tissue structure; reduces the deformation of brain cells and neurons, while inhibitor increased infarct injury. (A) Nissl staining at 7 and 14 days. Scale bars $=1,000 \mu \mathrm{m}$. (B) Nissl staining of the surviving cells at 7 days. Scale bars $=50 \mu \mathrm{m}$. (C) Cell morphology by hematoxylin and eosin staining at 7 days. Scale bars $=20 \mu \mathrm{m}$.

\section{Results}

Treadmill training attenuates neurological deficits, reduces infarct size and decreases the damage of brain tissue and deformation of neurons, while the inhibitor increases infarct injury

Treadmill training attenuates neurologic deficits. As shown in the Table II and Fig. $1 G$, the $\mathrm{S}$ group was normal (the scores=0). One day after MCAO, there were no significant differences in the scores of neurological impairments among the four groups $(\mathrm{P}>0.05)$ and the scores decreased gradually with the number of days. The score of the EM group was decreased compared with the $\mathrm{M}$ group in the same period (EM7 vs. M7: $\mathrm{P}<0.01$; EM14 vs. M14: $\mathrm{P}<0.01)$. The scores of the IM group were increased compared with the $\mathrm{M}$ group (IM7 vs. M7: $\mathrm{P}<0.05$; IM14 vs. M14: $\mathrm{P}<0.01)$. The score of the EM group was decreased compared with the IEM group (IEM7 vs. EM7: $\mathrm{P}<0.01$; IEM14 vs. EM14: P>0.05).

Treadmill training reduces the infarct volume. As shown in Fig. 1E and F, the white area represents damaged brain cells, and the red area represents functional cells. As expected, no infarction was observed in group S. TTC staining showed that the infarct volume in the EM group was smaller than that in the $\mathrm{M}$ group during the same period $(\mathrm{P}<0.01)$, while the infarct volume in the IM group was larger than that in the $M$ group during the same period $(\mathrm{P}<0.01)$. Compared with the EM group, the IEM group had larger infarct volumes (IEM7 vs. EM7: P<0.05; IEM14 vs. EM14: P<0.01) (Table III).

Treadmill training decreases the damage of brain tissue and deformation of neurons. As shown in Fig. 2, no infarction in the $\mathrm{S}$ group was found, but infarction was discovered in the ischemic areas of the other groups. In group $S$, as shown in Fig. 2, the neurons in the cerebral cortex were regular in shape and normal in structure. The nucleolus was clear. In the $\mathrm{M}$ and IM groups, numerous neurons in the ischemic penumbra were deformed and necrotic. Increased nuclear pyknosis and nuclear fragmentation occurred compared with in the $\mathrm{S}$ group. The Nissl bodies were narrowed, deeply stained and in some instances, disappeared. The cells were swollen, the surrounding space was enlarged and the shape was irregular. In the EM and IEM groups, the cells had a normalized arrangement, and a few cells were deformed and necrotic.

Treadmill training enhances the activation of Wnt/ $\beta$-catenin signaling pathway in the ischemic penumbra. Compared with group $\mathbf{M}$, the expression of Wnt3a and nuclear $\beta$-catenin protein in the EM group was increased (Wnt3a, EM7 vs. M7: $\mathrm{P}<0.01$; EM14 vs. M14: $\mathrm{P}<0.05$; nuclear $\beta$-catenin, EM7 vs. 
A

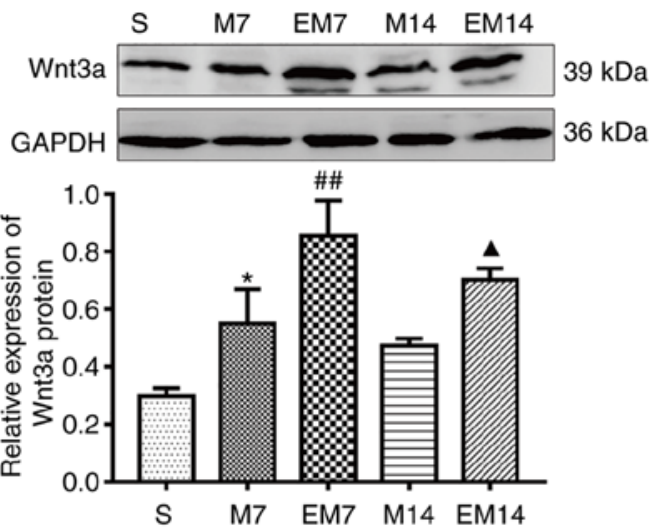

C
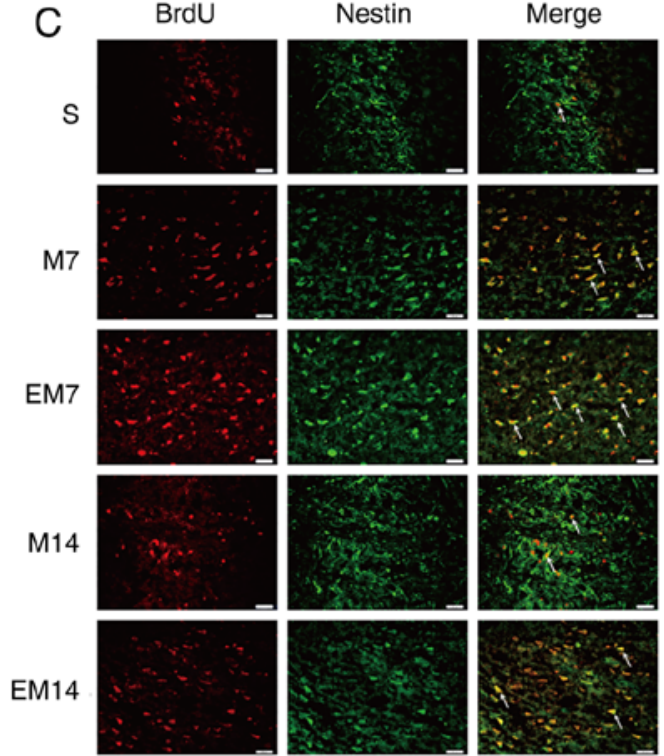

E
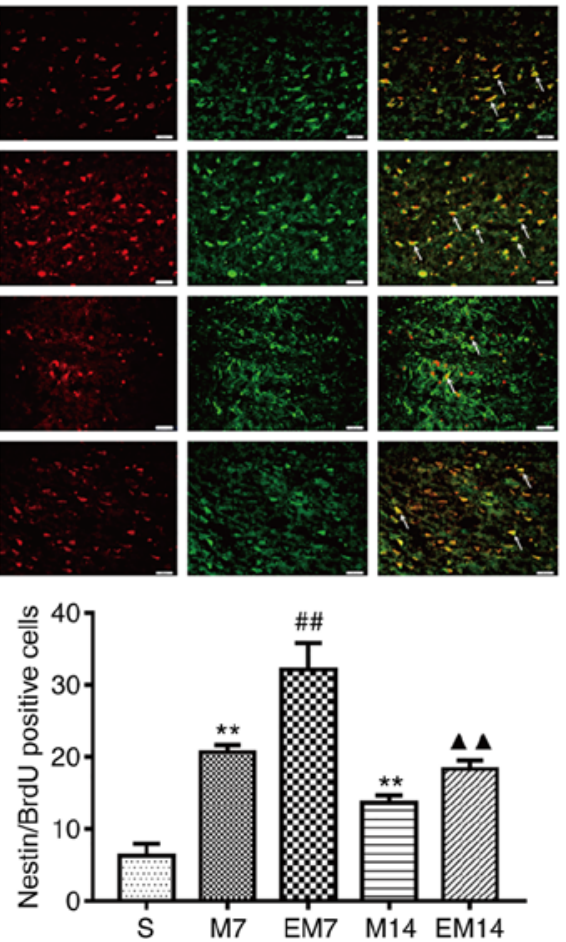

B
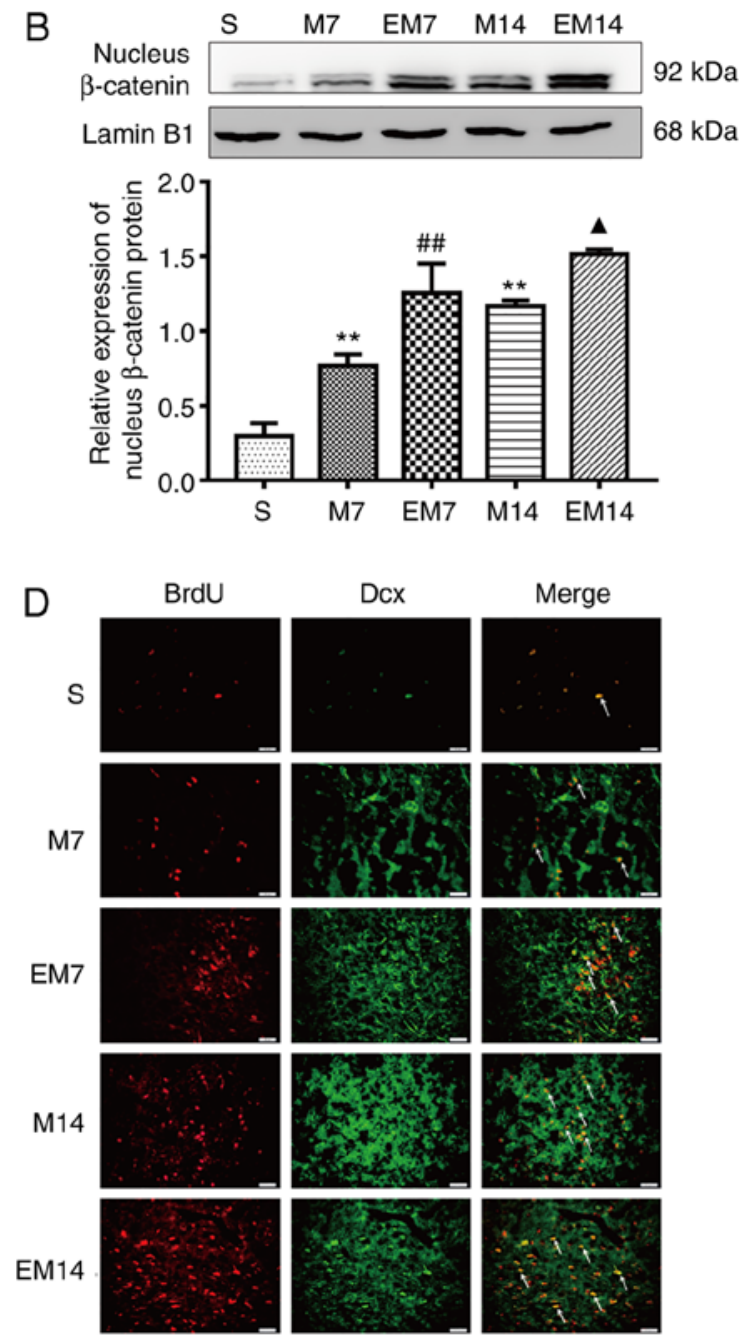

$\mathrm{F}$

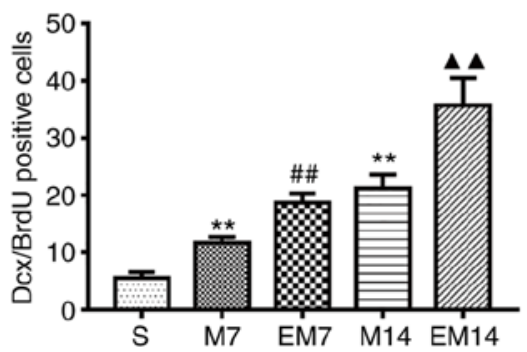

Figure 3. Treadmill training enhances the activation of the Wnt/ $\beta$-catenin signaling pathway and improves neurogenesis in the ischemic penumbra. (A) Protein expression levels and western blot analysis of Wnt3a. (B) Protein expression levels and western blot analysis of nucleus $\beta$-catenin. Data are presented as the mean \pm SD (n=3). (C) IF of Nestin/BrdU-positive cells in the ischemic penumbra. (D) IF of Dcx/BrdU-positive cells in the ischemic penumbra. (E) The number of Nestin/BrdU-positive cells in the ischemic penumbra. (F) The number of Dcx/BrdU-positive cells in the ischemic penumbra. Data are presented as the mean $\pm \mathrm{SD}(\mathrm{n}=6)$. Scale bars $=20 \mu \mathrm{m}$. ${ }^{*} \mathrm{P}<0.05$ and ${ }^{* *} \mathrm{P}<0.01$ vs. the $\mathrm{S}$ group; ${ }^{\# \#} \mathrm{P}<0.01$ vs. the M7 group; ${ }^{\mathbf{\Delta}} \mathrm{P}<0.05$ and ${ }^{\boldsymbol{\Delta}} \mathrm{P}<0.01$ vs. the $\mathrm{M} 14$ group. $\mathrm{SD}$, standard deviation; BrdU, bromodeoxyuridine; IF, immunofluorescence.

M7: P<0.01; EM14 vs. M14: P<0.05; Fig. 3A and B). Compared with group $\mathrm{S}$, the expression of $\mathrm{Wnt} 3 \mathrm{a}$ and nuclear $\beta$-catenin protein in the $\mathrm{M}$ group was increased (Wnt3a, $\mathrm{S}$ vs. $\mathrm{M} 7: \mathrm{P}<0.05$; $\mathrm{S}$ vs. $\mathrm{M} 14$ : $\mathrm{P}>0.05$; nuclear $\beta$-catenin, $\mathrm{S}$ vs. $\mathrm{M7}$ : $\mathrm{P}<0.01$; $\mathrm{S}$ vs. M14: $\mathrm{P}<0.01$; Fig. 3A and B)

Treadmill training increases the expression level of BDNF in the ischemic penumbra. The expression of BDNF protein and the number of BDNF-positive cells in the M groups was increased compared with in the $\mathrm{S}$ groups (western blotting: BDNF, S vs. M7: P<0.05; S vs. M14: P>0.05; immunofluores- cence: BDNF, S vs. M7: P<0.01; S vs. M14: P<0.01). Similarly, the expression of the BDNF protein and the number of BDNF-positive cells in EM group were increased compared with in the $\mathrm{M}$ group (western blotting: BDNF, EM7 vs. M7: $\mathrm{P}<0.01$; EM14 vs. M14: $\mathrm{P}<0.05$; immunofluorescence: BDNF, EM7 vs. M7: $\mathrm{P}<0.01$; EM14 vs. M14: $\mathrm{P}<0.01$; Fig. 4A-C).

Treadmill training improves regenerative neurogenesis in the ischemic penumbra. Nestin/BrdU is a specific marker of proliferating NSCs. Dcx/BrdU is a specific marker of proliferating neuroblasts and differentiating neurons (14). There were 

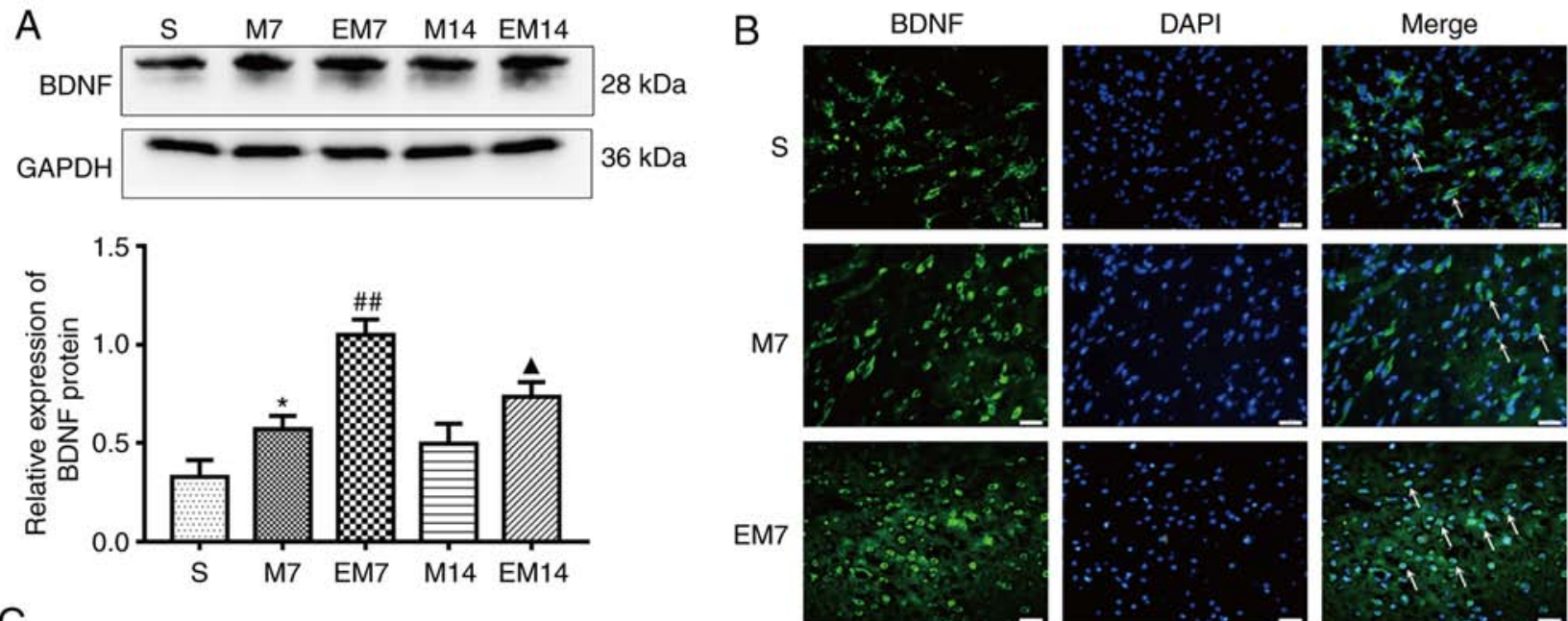

C
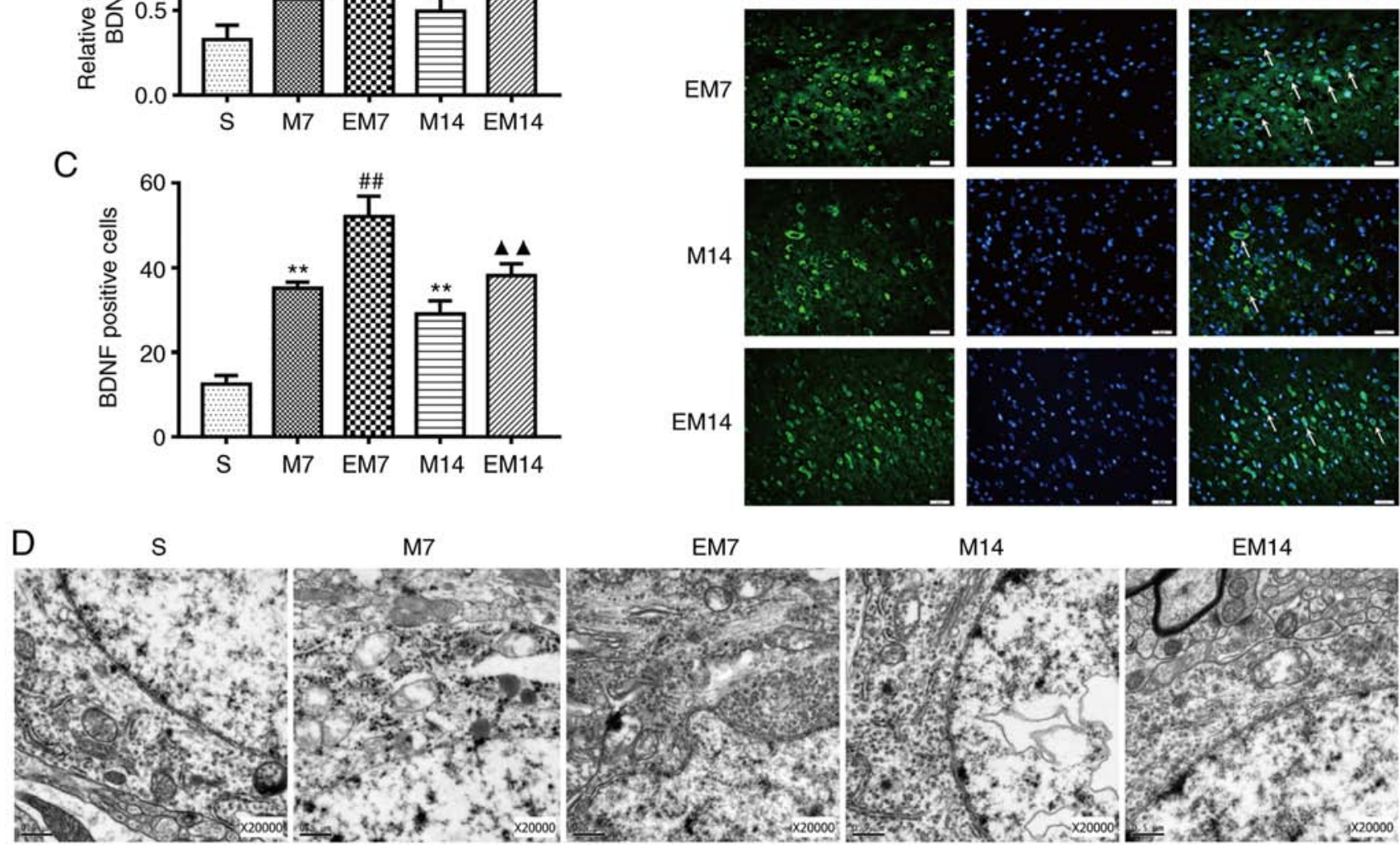

Figure 4. Treadmill training increases the expression level of BDNF and improves the morphology of cortical neurons in the ischemic penumbra after MCAO. (A) Protein expression levels and western blot analysis of BDNF. Data are presented as the mean $\pm \mathrm{SD}(\mathrm{n}=3)$. (B) Immunofluorescence of BDNF-positive cells in the ischemic penumbra. Scale bars $=20 \mu \mathrm{m}$. (C) The number of BDNF-positive cells in the ischemic penumbra. Data are presented as the mean \pm SD ( $\mathrm{n}=6$ ). (D) Transmission electron microscopy showed the neuron nucleus structures $(\mathrm{n}=3)$. Scale bars $=0.5 \mu \mathrm{m}$. ${ }^{*} \mathrm{P}<0.05$ and ${ }^{* * *} \mathrm{P}<0.01$ vs. the $\mathrm{S}$ group; ${ }^{\# \#} \mathrm{P}<0.01$ vs. the M7 group; ${ }^{\boldsymbol{\Lambda}} \mathrm{P}<0.05$ and ${ }^{\boldsymbol{\Lambda}} \mathrm{P}<0.01$ vs. the M14 group. S group, sham operation group; M group, model group; MCAO, middle cerebral artery occlusion; $\mathrm{SD}$, standard deviation; BDNF, brain derived neurotrophic protein; DAPI, 4,6-diamidino-2-phenylindole.

almost no Dex/BrdU-positive cells and Nestin/BrdU-positive cells in the ischemic penumbra of the $\mathrm{S}$ group in this study. Compared with that of the sham group, the number of Nestin/BrdU-positive cells in the MCAO group increased, peaked at 7 days and then decreased at 14 days $(\mathrm{P}<0.01$ vs. sham). Similarly, compared with that of the M group, the number of Nestin/BrdU-positive cells in the EM group further increased and the number of cells in the EM group peaked at 7 days and showed a downward trend at 14 days (EM7 vs. M7: $\mathrm{P}<0.01$; EM14 vs. M14: $\mathrm{P}<0.01$; Fig. $3 \mathrm{C}$ and E). The number of the Dcx/BrdU-positive cells reached a peak at 14 days in the $\mathrm{M}$ group and EM group. The Dcx/BrdU-positive cells in group $\mathrm{M}$ were significantly increased compared with that of the sham operation group $(\mathrm{P}<0.01$ vs. sham). Similarly, on the 7 and 14th days, the Dcx/BrdU-positive cells in the EM group were significantly increased compared with in the $M$ group (EM7 vs. M7: P<0.01; EM14 vs. M14: P<0.01; Fig. 3D and F).
The present study used TEM to observe the ultrastructure of the neuron nucleus (Fig. 4D). In the specimens of group S, it was observed that the distribution of nuclear chromatin was uniform, the double nuclear membrane of the neuron was clear and complete. TEM analysis showed that compared with those of group S, the chromatin distribution of group $\mathrm{M}$ was irregular, the nuclear membrane structure was blurred and the mitochondrial crest was deformed. In the EM group, the damage of nucleus was alleviated and the contraction of nuclear membrane was not obvious. The edge of the nucleus was smooth and a few of the inner cristae of mitochondria were broken.

Treadmill training increases the repair of myelin in the ischemic penumbra. To investigate the effect of treadmill exercise on myelin repair, the expression level of the myelin marker MBP was analyzed in the ischemic penumbra. 

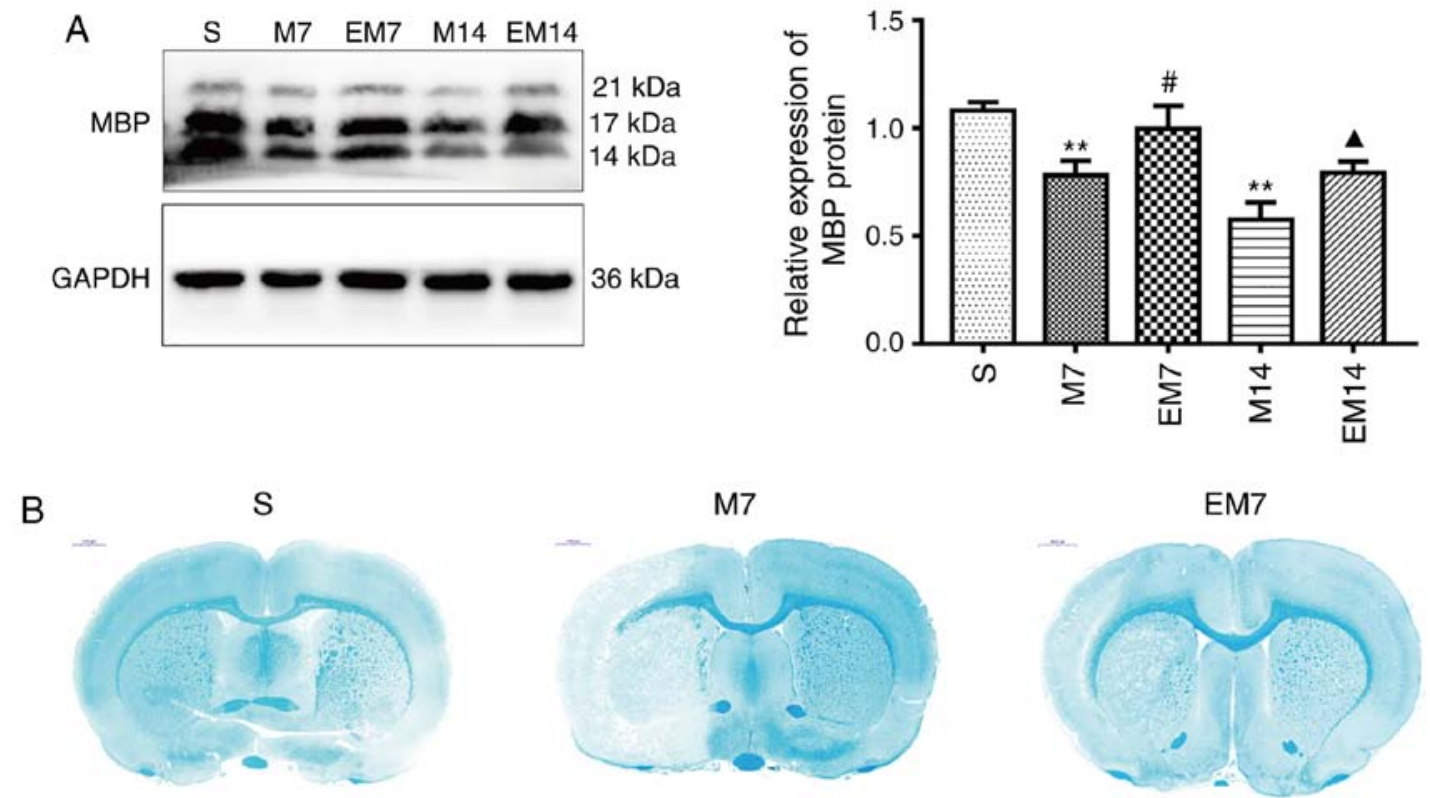

M7
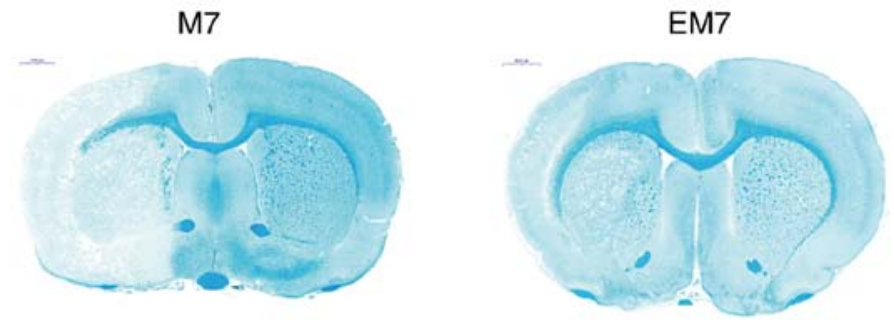

M14

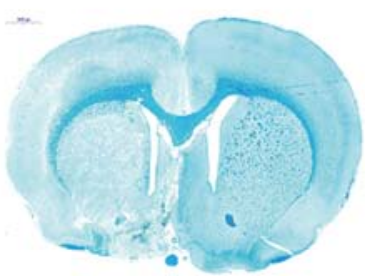

EM14

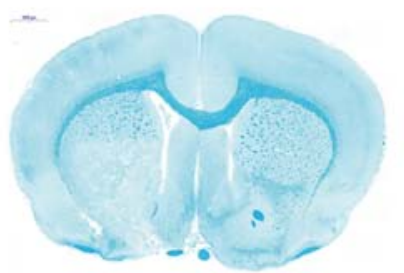

Figure 5. Treadmill training increases the repair of myelin in the ischemic penumbra. (A) Protein expression levels and western blot analysis of MBP. Data are presented as the mean \pm standard deviation $(\mathrm{n}=3)$. (B) LFB staining in the ischemic penumbra after middle cerebral artery occlusion. $\mathrm{Scale}$ bars $=1,000 \mu$ m. ${ }^{* *} \mathrm{P}<0.01$ vs. the $\mathrm{S}$ group; ${ }^{\#} \mathrm{P}<0.05$ vs. the $\mathrm{M} 7$ group; ${ }^{\wedge} \mathrm{P}<0.05$ vs. the $\mathrm{M} 14$ group. $\mathrm{S}$ group, sham operation group; $\mathrm{M}$ group, model group; MBP, myelin basic protein.

As shown in Fig. 5A, western blot analysis showed that the expression of MBP in the $\mathrm{M}$ group was significantly decreased compared with the $\mathrm{S}$ group $(\mathrm{P}<0.01)$. At 7 or 14 days after $\mathrm{MCAO}$, compared with $\mathrm{M}$ group, the expression of MBP protein in EM group was increased (EM7 vs. M7: $\mathrm{P}<0.05$; EM14 vs. M14: $\mathrm{P}<0.05)$. In sham brain sections, the myelinated fibers were strongly stained by LFB, the myelinated fibers were dense and uniform, and the morphology of the myelinated fibers was complete. After 7 and 14 days of $\mathrm{MCAO}$, all groups, except for the $\mathrm{S}$ group, showed lesions in the cortex and subcortex. In the $\mathrm{M}$ group, LFB staining analysis showed significant demyelination in the ischemic penumbra. Demyelination was significantly decreased in the EM group compared with the group $\mathrm{M}$ during the same period (Fig. 5B).

Inhibitor abolishes neurogenesis in the ischemic penumbra. After the addition of the $\mathrm{Wnt} / \beta$-catenin pathway inhibitor XAV939, compared with the $M$ group, the protein expression of Wnt3a and nuclear $\beta$-catenin in the IM group was decreased. (Wnt3a, IM7 vs. M7: P<0.01; IM14 vs. M14: $\mathrm{P}<0.01$; nuclear $\beta$-catenin, IM7 vs. M7: $\mathrm{P}<0.01$; IM14 vs. M14: $\mathrm{P}<0.01$; Fig. 6A and B).Compared with the $\mathrm{M}$ group, the expression of the BDNF protein in the IM group was decreased during the same period (IM7 vs. M7: P<0.01; IM14 vs. M14: P<0.01; Fig. 7A).
The same trend was observed in the BDNF-positive cells (IM7 vs. M7: P<0.01; IM14 vs. M14: P<0.01; Fig. 7B and C). Compared with the M7 group, the number of Dcx/BrdU positive cells in the IM7 group had no significant change, while there was a decrease in the number of Dcx/BrdU-positive cells between group M14 and group IM14 (Dcx/BrdU, IM7 vs. M7: P>0.05; IM14 vs. M14: $\mathrm{P}<0.05$; Fig. $6 \mathrm{D}$ and F). There was a difference in the number of Nestin/BrdU-positive cells between group M7 and group IM7. Compared with the M14 group, the number of Nestin/BrdU-positive cells in the IM14 group had no significant change (IM7 vs. M7: P<0.01; IM14 vs. M14: P>0.05; Fig. 6C and E).

In terms of neuronal structure, TEM showed that compared with group M, the IM group showed dissolved cytoplasm, coacervation of nuclear chromatin and a shrunken or dissolved nuclear membrane. Most of the organelles disappeared and only a small number of denatured mitochondria (with focal cavitation) were present (Fig. 7D).

Inhibitor abolishes myelin repair in the ischemic penumbra. The expression of MBP and the degree of demyelination at 7 and 14 days after injection of the inhibitor was further examined. Compared with group $\mathrm{M}$, the expression of MBP protein in the IM group was decreased (IM7 vs. M7: P<0.01; IM14 vs. M14: $\mathrm{P}<0.01$; Fig. 8A). LFB staining showed that compared 
A
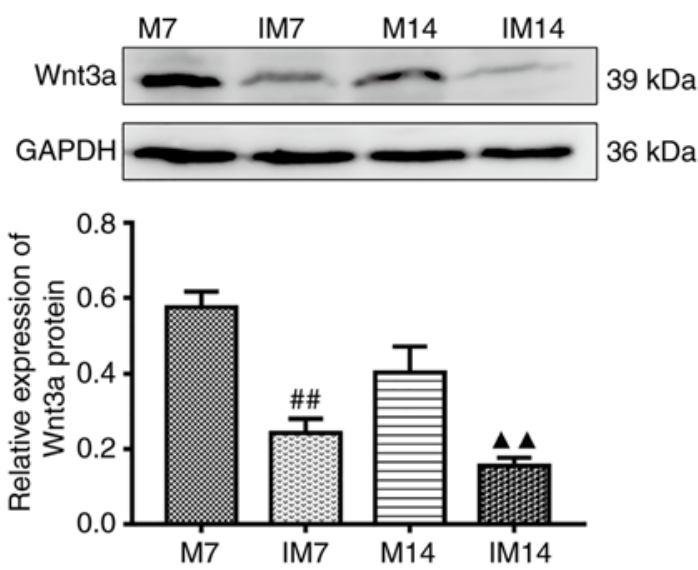

C
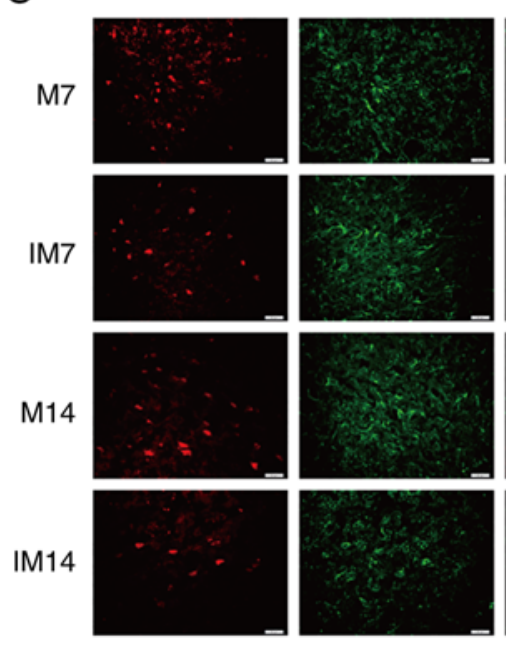

$\mathrm{E}$

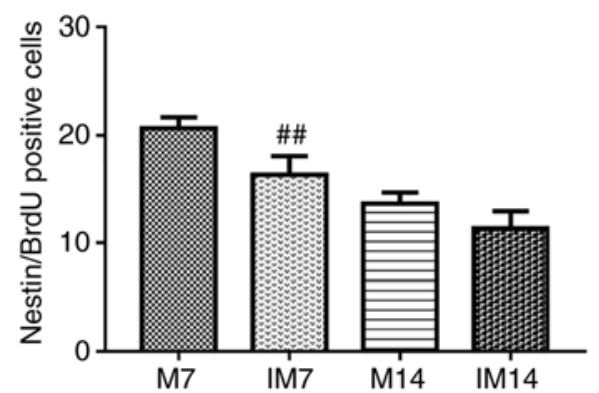

B
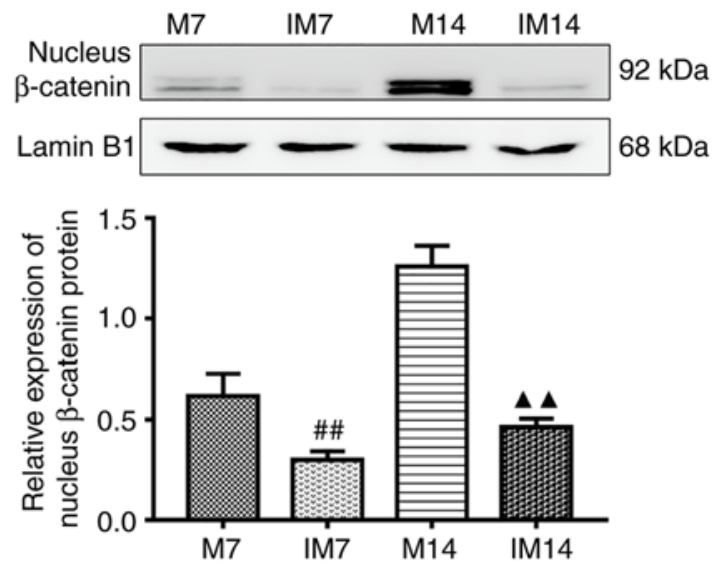

D

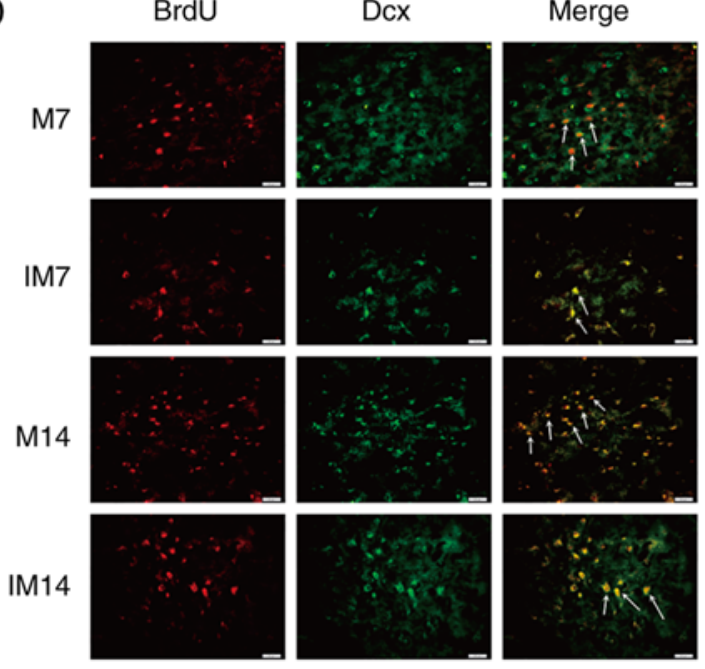

$\mathrm{F}$

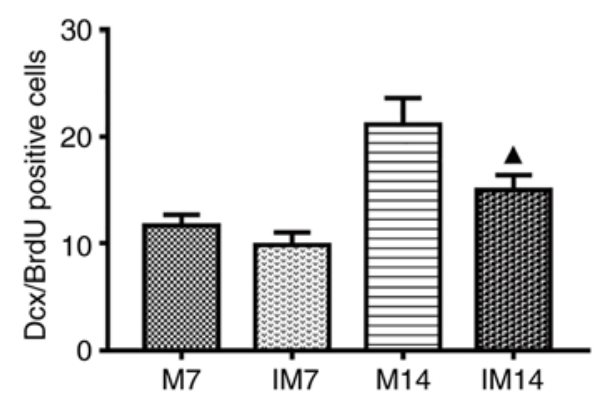

Figure 6. Inhibition of the Wnt/ $\beta$-catenin pathway abolishes neurogenesis in the ischemic penumbra. (A) Protein expression levels and western blot analysis of Wnt3a. (B) Protein expression levels and western blot analysis of nucleus $\beta$-catenin. Data are presented as the mean \pm SD ( $n=3$ ). (C) IF of Nestin/BrdU-positive cells in the ischemic penumbra. (D) IF of Dcx/BrdU-positive cells in the ischemic penumbra. (E) The number of Nestin/BrdU-positive cells in the ischemic penumbra. (F) The number of Dcx/BrdU-positive cells in the ischemic penumbra. Data are presented as the mean $\pm \mathrm{SD}(\mathrm{n}=6)$. Scale bars $=20 \mu \mathrm{m}$. ${ }^{\# \#} \mathrm{P}<0.01 \mathrm{vs}$. the M7 group; ${ }^{\wedge} \mathrm{P}<0.05$ and ${ }^{\Delta \boldsymbol{\Delta}} \mathrm{P}<0.01$ vs. the M14 group. M group, model group; SD, standard deviation; IF, immunofluorescence; BrdU, bromodeoxyuridine.

with group $\mathrm{M}$, the integrity of the myelin sheath was decreased and demyelination was increased in group IM (Fig. 8B).

Inhibitor suppresses exercise-promoted neurogenesis in the ischemic penumbra. After 7 and 14 days of MCAO, four groups of EM7, IEM7, EM14 and IEM14 were designed to prove whether the Wnt/ $\beta$-catenin signaling pathway was involved in exercise-promoted neurogenesis. The results showed that compared with group EM, the expression of Wnt3a and nucleus $\beta$-catenin protein in group IEM were decreased $(\mathrm{Wnt} 3 \mathrm{a}$, IEM7 vs. EM7: P<0.01; IEM14 vs. EM14: $\mathrm{P}<0.05$; nucleus $\beta$-catenin, IEM7 vs. EM7: $\mathrm{P}<0.01$; IEM14 vs. EM14: $\mathrm{P}<0.01$; Fig. 9A and B). During the same period, compared with group EM, the expression of BDNF protein and the number of BDNF positive cells in group IEM were decreased (western blotting: IEM7 vs. EM7: P<0.01; IEM14 vs. EM14: P<0.05; immunofluorescence: IEM7 vs. EM7: P<0.01; IEM14 vs. EM14: $\mathrm{P}<0.01$; Fig. 10A-C). At 7 and 14 days after MCAO, the expression levels of Dcx/BrdU-and Nestin/BrdU-positive cells in the EM group were different from those in the IEM group (Fig. 9C-F). At 7 days, there was a significant difference in the number of Dcx/BrdU-positive cells between IEM7 and EM7. However, at 
A
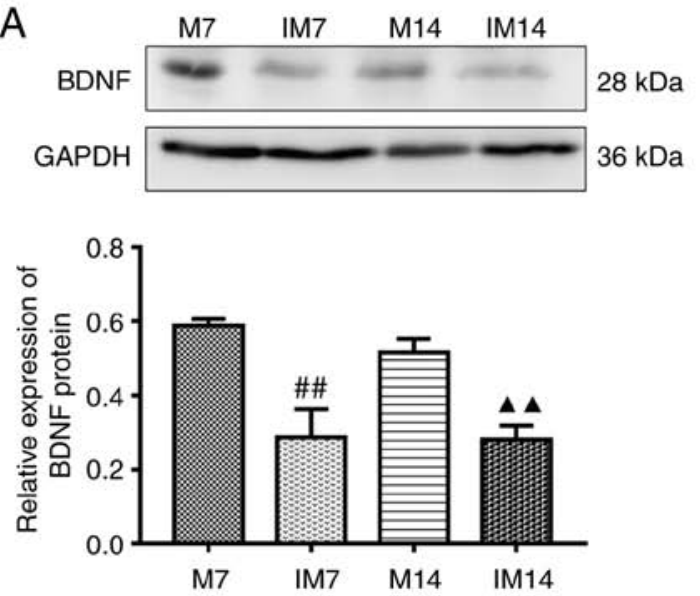

C

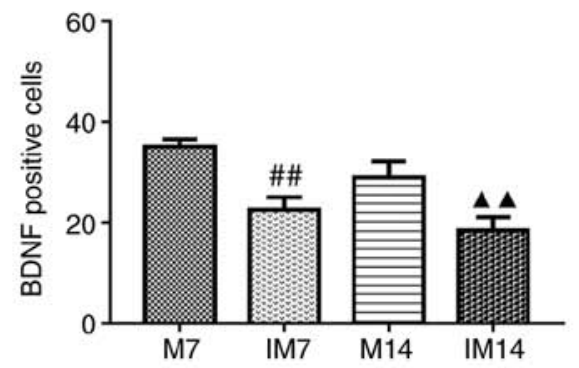

D

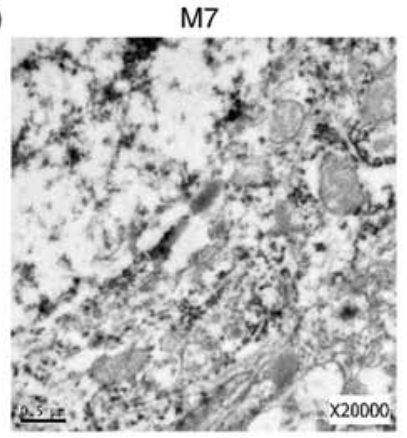

B
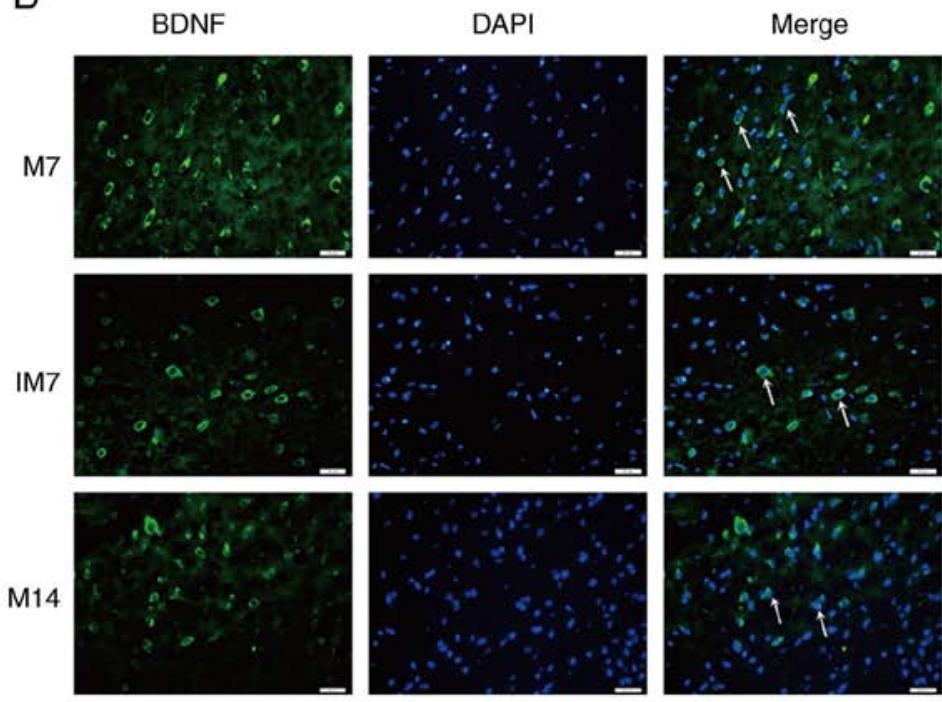

IM14

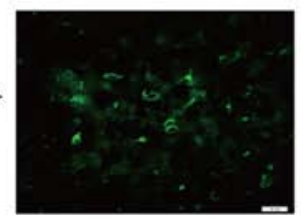

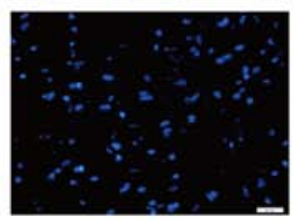

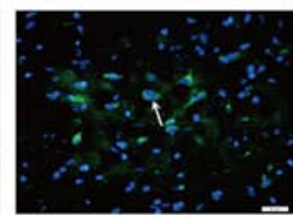

IM7

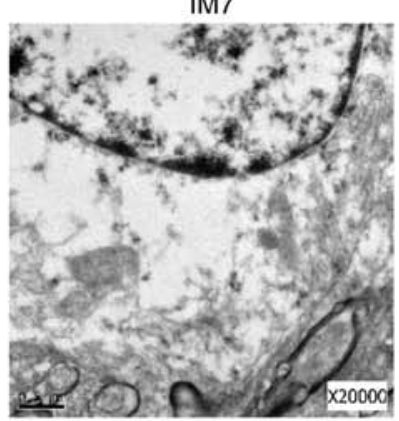

M14

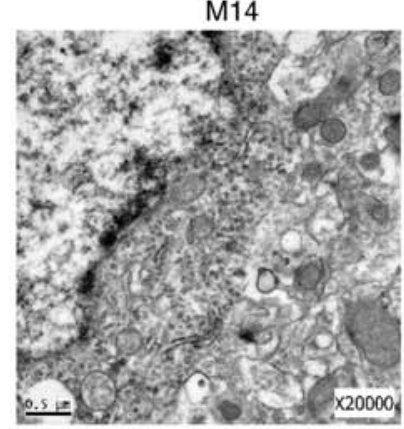

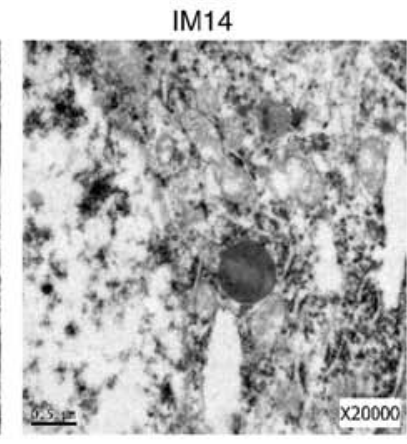

Figure 7. Inhibitor downregulates BDNF expression and damages the morphology of cortical neurons in the ischemic penumbra after middle cerebral artery occlusion. (A) Protein expression levels and western blot analysis of BDNF. Data are presented as the mean \pm SD ( $\mathrm{n}=3$ ). (B) Immunofluorescence of BDNF-positive cells in the ischemic penumbra. Scale bars $=20 \mu \mathrm{m}$. (C) The number of BDNF-positive cells in the ischemic penumbra. Data are presented as the mean $\pm \mathrm{SD}(\mathrm{n}=6)$. (D) Transmission electron microscopy showed the neuron nucleus structures $(\mathrm{n}=3)$. Scale bars $=0.5 \mu \mathrm{m}$. ${ }^{\# \#} \mathrm{P}<0.01 \mathrm{vs}$. the $\mathrm{M} 7 \mathrm{group}$; $\triangle{ }^{\wedge} \mathrm{P}<0.01$ vs. the M14 group. M group, model group; SD, standard deviation; BDNF, brain derived neurotrophic factor; DAPI, 4,6-diamidino-2-phenylindole.

14 days, there was no difference in the IEM14 group compared with the EM14 group. Similarly, there was no significant difference in the number of Nestin/BrdU-positive cells between IEM14 and EM14. However, at 7 days, the number of positive cells in the IEM7 group was reduced compared with the EM7 group (Dcx/BrdU, IEM7 vs. EM7: P<0.01; IEM14 vs. EM14: P>0.05; Nestin/BrdU, IEM7 vs. EM7: P<0.01; IEM14 vs. EM14: P>0.05).

By observing the ultrastructure of neuronal nuclei under the TEM, the present study found that the IEM group showed edema in the cytoplasm of the neurons, mitochondrial swelling, mitochondrial crest deformation and organelle reduction compared with the EM group (Fig. 10D).

Inhibitor abolishes exercise-promoted myelin repair in the ischemic penumbra. The current study further detected the expression of MBP and the changes in LFB staining at 7 or 14 days in group EM7, IEM7, EM14 and IEM14. Compared with group EM, the expression of MBP protein in group IEM was decreased (IEM7 vs. EM7: P<0.05; IEM14 vs. EM14: $\mathrm{P}<0.01$; Fig. 11A). The results of LFB staining showed that compared with those of the EM group, the myelin staining in the IEM group became lighter, the integrity became worse and the degree of demyelination increased (Fig. 11B).

\section{Discussion}

The authors' previous studies have shown that treadmill exercise can promote angiogenesis and neurogenesis in the ischemic penumbra of adult rats. In addition, treadmill exercise can promote dendritic modification and synaptic plasticity $(25,29)$. In this study, the mechanism of neurogenesis and myelin repair after ischemic stroke in juvenile rats was focused on. The results showed that treadmill exercise can promote neurogenesis and myelin repair after ischemic stroke in juvenile rats. In addition, the $\mathrm{Wnt} / \beta$-catenin signaling pathway 

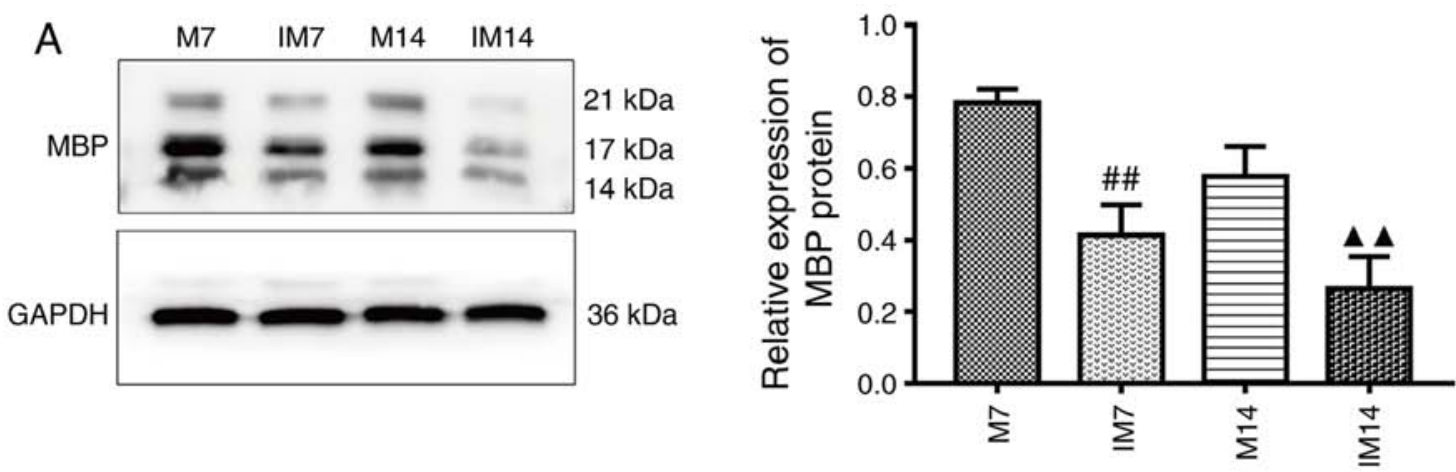

B

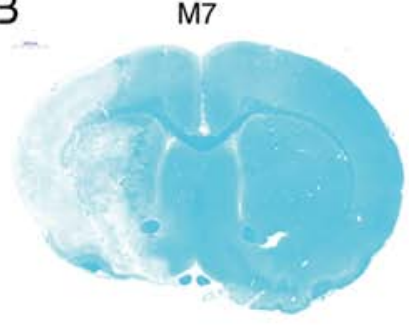

IM7

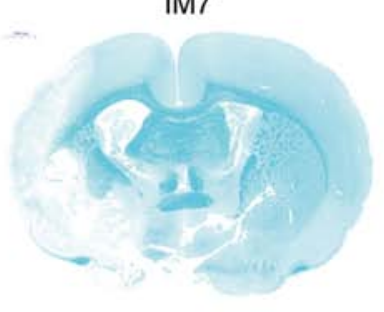

M14

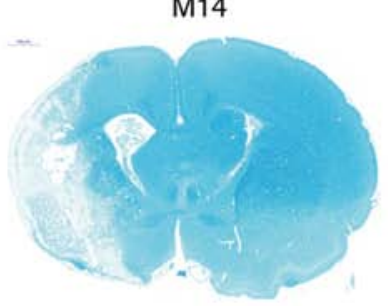

IM14

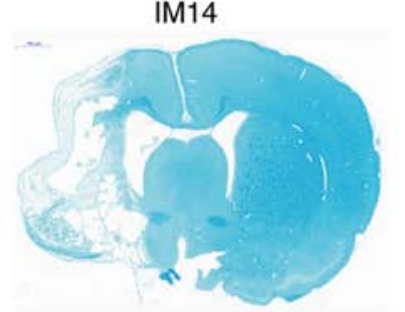

Figure 8. Inhibition of the Wnt/ $\beta$-catenin pathway abolishes myelin repair in the ischemic penumbra. (A) Protein expression levels and western blot analysis of MBP. Data are presented as the mean \pm SD $(n=3)$. (B) Luxol fast blue staining in the ischemic penumbra after middle cerebral artery occlusion. ${ }^{\#}$ P $<0.05$ vs. the M7 group; ${ }^{\wedge}{ }^{\wedge} \mathrm{P}<0.01$ vs. the M14 group. M group, model group; MCAO, middle cerebral artery occlusion; MBP, myelin basic protein; SD, standard deviation.

was involved in neurogenesis and myelin repair stimulated by treadmill training.

For a long time, the focus of research has been on the use of neurogenesis to promote neuronal replacement and enhance endogenous brain repair. However, studies have found that only a very small number of neurons survive in neurogenesis after adult ischemic stroke (39). The latest research shows that after cerebral ischemia, significant reaggregation of neurons in the juvenile striatum occurs, indicating that the juvenile brain has the ability to repair itself (10). In the present study, Nestin/BrdU- and Dcx/BrdU-positive cells increased in group $\mathrm{M}$ at 7 and 14 days after MCAO. Nestin/BrdU-positive cells reached the peak on the 7th day and then decreased gradually. The number of Dcx/BrdU-positive cells increased throughout and reached its peak on the 14th day. In addition, the expression levels of Wnt3a, nuclear $\beta$-catenin and BDNF increased on the 7 and 14th days after MCAO. Physical exercise has a neuroprotective effect on both human and animals. A previous study has shown that pre-ischemic treadmill training reduces cerebral infarction volume, brain edema and neurological deficit and improves brain injury after ischemic stroke (40). The authors' previous studies have shown that after ischemic stroke in adult rats or mice, treadmill exercise enhanced neurogenesis by promoting proliferation, migration and differentiation into mature neurons of NPCs from the subventricular area to the ischemic penumbra $(29,30)$. In the present study, it was found that treadmill exercise promoted the expression of Nestin/BrdU- and Dcx/BrdU-positive cells in the ischemic penumbra of juvenile rats. It is suggested that treadmill exercise enhances neurogenesis by promoting the increment and migration of neuroblasts. More importantly, how to stimulate the neurogenesis of the penumbra and explore its mechanism is the focus of the current research.
After neonatal hypoxia-ischemia injury, treadmill exercise helps improve the recovery of behavior after hypoxia-ischemia via the upregulation of myelin components and neurogenesis (41). In addition, treadmill training combined therapy may improve motor and memory function by increasing the oligodendroglia involved in the cyclic AMP-responsive element-binding protein/BDNF signaling pathway, to restore the myelin components of neonates after hypoxia-ischemia (13). A study has identified that long-term exercise can promote the expression of BDNF and improve the myelin damage caused by ischemia (28). It was found that NPCs could proliferate and differentiate into oligodendrocyte progenitor cells (OPCs) after cerebral ischemia. OPCs can differentiate into mature oligodendrocytes to repair damaged myelin sheaths (42). Oligodendrocyte injury and demyelination can lead to neurological functional deficits (43-45). A previous study has shown that exercise can restore ischemia-induced myelinated hippocampal nerve fiber injury (28). In the present study, treadmill exercise promoted the expression of MBP in the penumbra and reduced demyelination. In this study, treadmill exercise may alleviate brain injury after stroke by promoting neurogenesis and myelin repair. However, the mechanism of brain protection by treadmill training remains to be studied.

A previous study has shown that there is a pathological decrease in Wnt activity in subventricular zone (SVZ) after ischemic stroke (19). More importantly, administration of exogenous Wnt3a can promote the migration and differentiation of newborn neuroblasts to the ischemic cortex (19). It was found that the increased expression of $\beta$-catenin could regulate Wnt3a (46). Moreover, it was found that the Wnt pathway is directly involved in the upregulation of BDNF, indicating that the Wnt/ $\beta$-catenin signaling pathway is one of the upstream pathways of BDNF (19). BDNF plays an important role in brain 
A
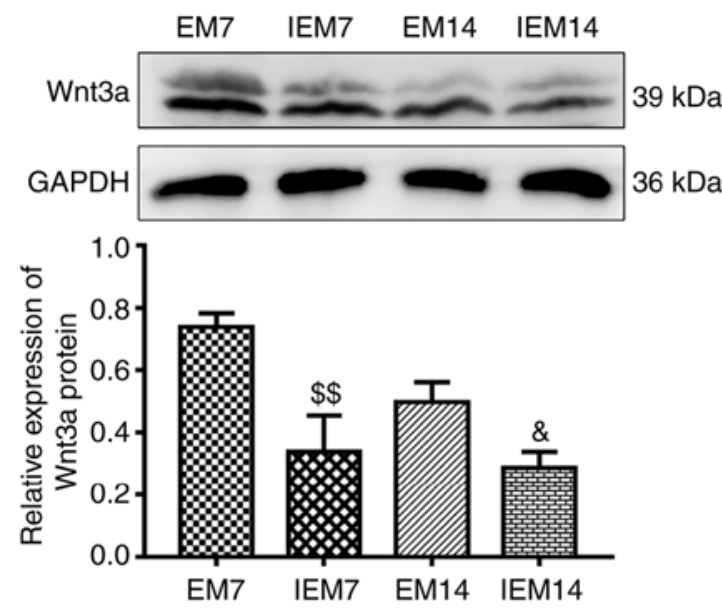

C
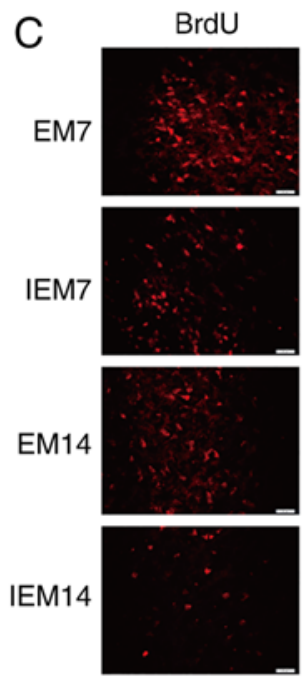

E

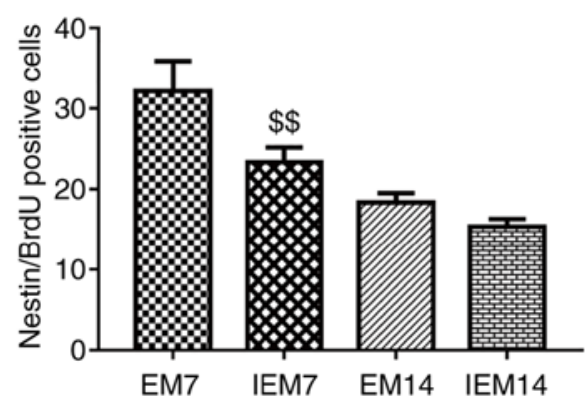

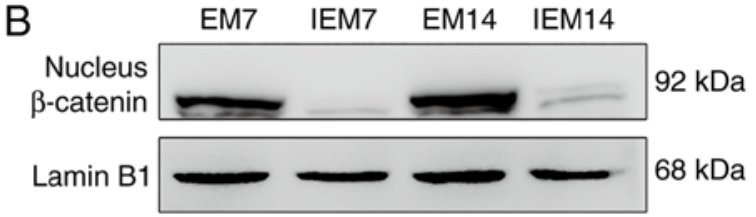
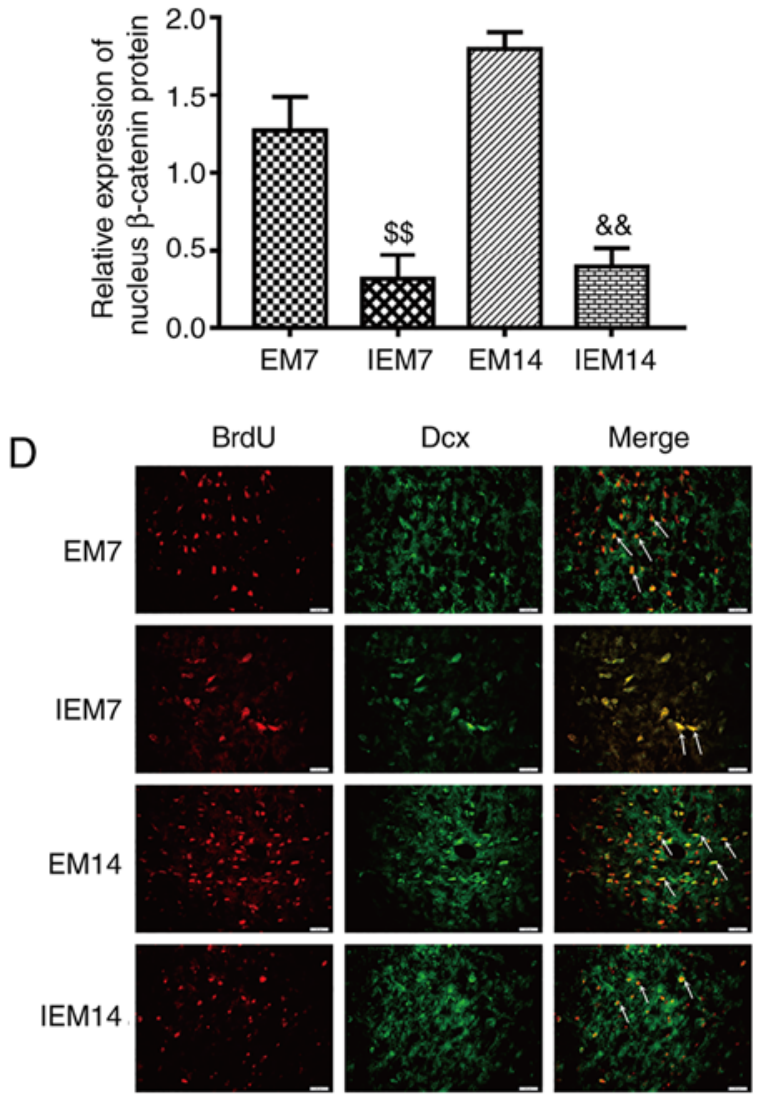

$\mathrm{F}$

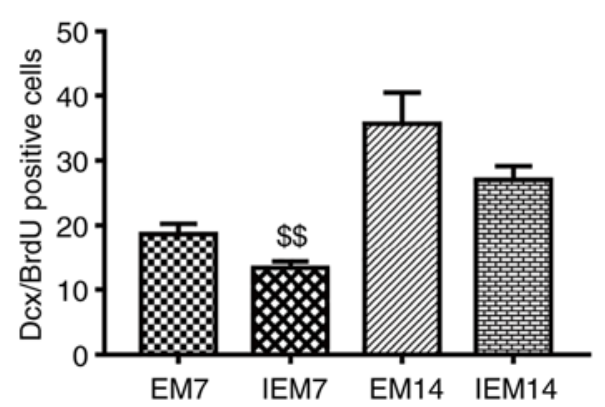

Figure 9. Inhibition of the Wnt/ $\beta$-catenin signaling pathway suppresses exercise-promoted neurogenesis in the ischemic penumbra. (A) Protein expression levels and western blot analysis of Wnt3a. (B) Protein expression levels and western blot analysis of nucleus $\beta$-catenin. Data are presented as the mean \pm SD $(n=3)$. (C) IF of Nestin/BrdU-positive cells in the ischemic penumbra. (D) IF of Dcx/BrdU-positive cells in the ischemic penumbra. (E) The number of Nestin/ BrdU-positive cells in the ischemic penumbra. (F) The number of Dcx/BrdU-positive cells in the ischemic penumbra. Data are presented as the mean \pm SD $(\mathrm{n}=6)$. Scale bars $=20 \mu \mathrm{m} .{ }^{\$ \$} \mathrm{P}<0.01 \mathrm{vs}$. the EM7 group; ${ }^{\&} \mathrm{P}<0.05$ and ${ }^{\&}{ }^{\mathrm{P}}<0.01$ vs. the EM14 group. EM group, treadmill training model group; IF, immunofluorescence; BrdU, bromodeoxyuridine.

development and brain injury. BDNF can promote neurogenesis, improve synaptic plasticity, and nourish neurons $(47,48)$. Moreover, BDNF is released by neurons and secreted mainly through dendrites (49-51). It is reported that exogenous BDNF injection can promote neuronal regeneration, while the loss of BDNF inhibited the differentiation of intermediate neurons in mice (52-54). It has been found that activation of the Wnt signaling pathway in SVZ can promote endogenous neurogenesis, increase the number of new neurons and improve nerve function after injury (19). A previous study has shown that neurogenesis in the adult brain may contribute not only to brain repair but also to overall plasticity (55). Interestingly, in this research, it was found that neurogenesis after ischemic stroke in juvenile rats also promotes potential brain repair. Consistent with a previous study (29), at 7 and 14 days after treadmill exercise in juvenile MCAO rats, numerous newborn neuroblasts in the ischemic penumbra were observed. Moreover, an increase in Wnt3a, nucleus $\beta$-catenin and BDNF protein levels and BDNF-positive cells were found in the EM7 and EM14 groups. 
A
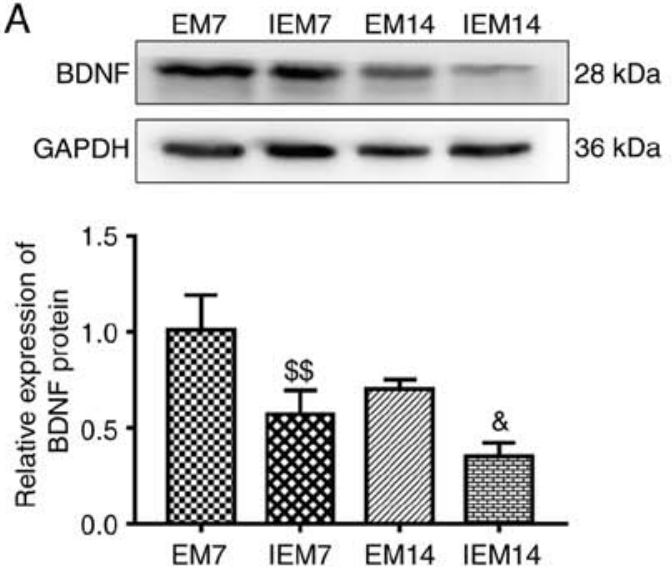

C

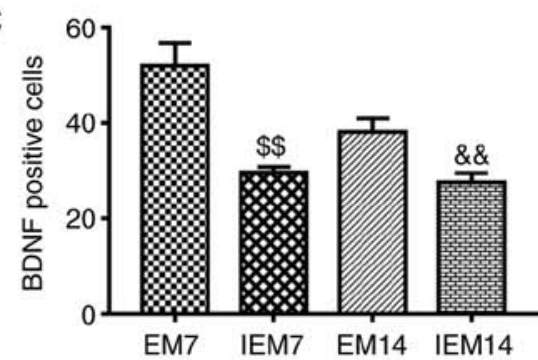

B

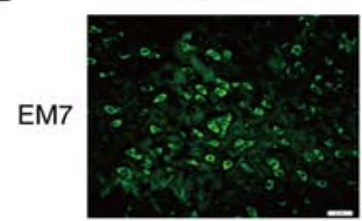

IEM7
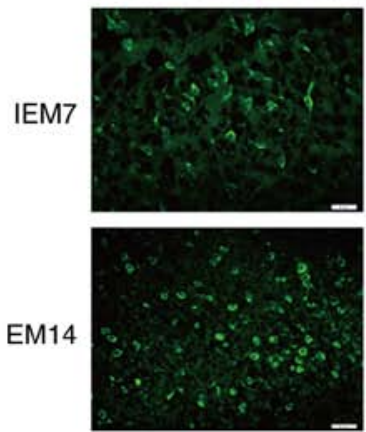

IEM14

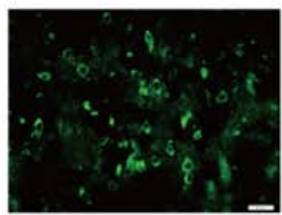

DAPI
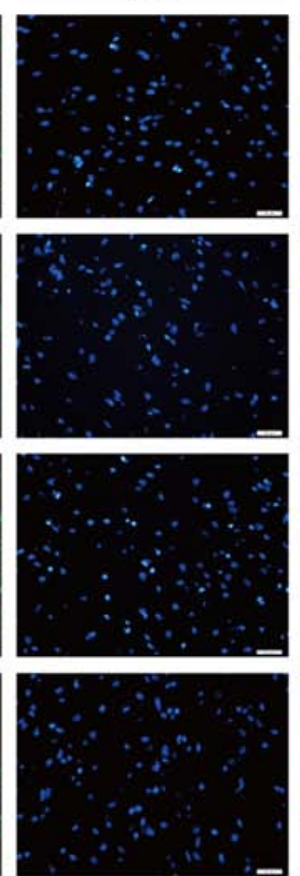

Merge
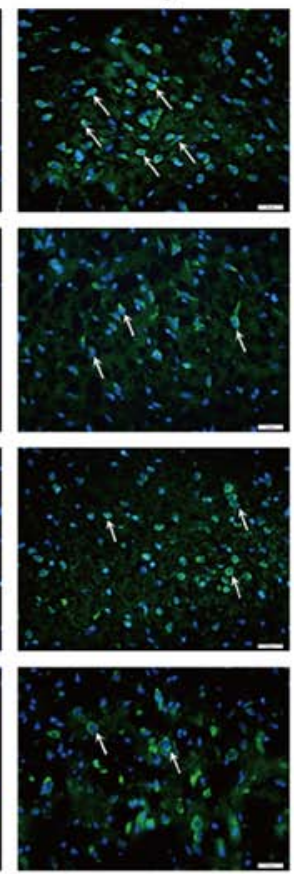

D

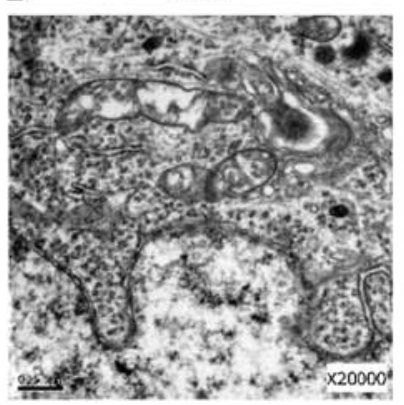

IEM7

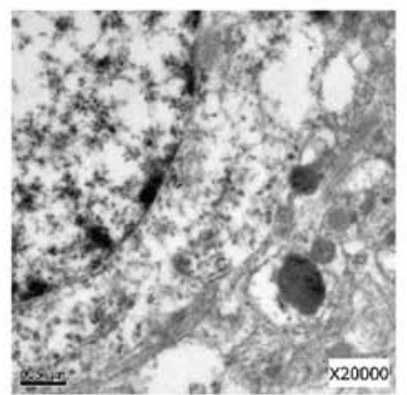

EM14

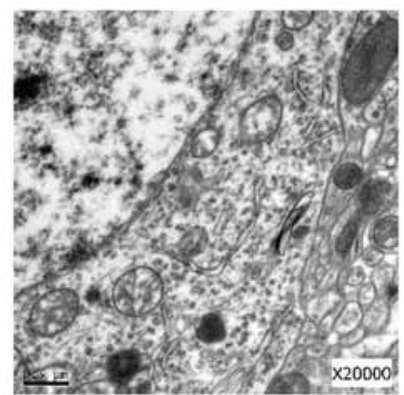

IEM14

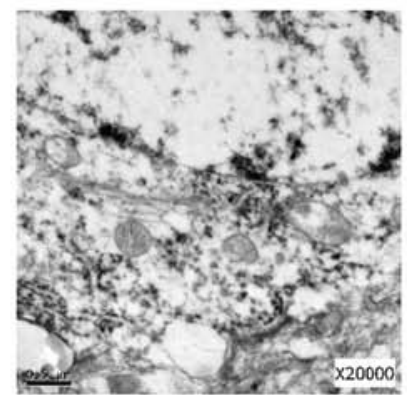

Figure 10. Inhibition of the Wnt/ $\beta$-catenin signaling pathway abolishes exercise-promoted BDNF expression and the morphology of cortical neurons in the ischemic penumbra after middle cerebral artery occlusion. (A) Protein expression levels and western blot analysis of BDNF. Data are presented as the mean \pm SD ( $=3$ ). (B) Immunofluorescence of BDNF-positive cells in the ischemic penumbra. Scale bars $=20 \mu \mathrm{m}$. (C) The number of BDNF-positive cells in the ischemic penumbra. Data are presented as the mean \pm SD $(n=6)$. (D) Transmission electron microscopy showed the neuron nucleus structures ( $n=3)$. Scale bars $=0.5 \mu \mathrm{m} .{ }^{\$ \$} \mathrm{P}<0.05$ vs. the EM7 group; ${ }^{\&} \mathrm{P}<0.05$ and ${ }^{\& \&} \mathrm{P}<0.01$ vs. the EM14 group. EM group, treadmill training model group. BDNF, brain derived natriuretic factor; SD, standard deviation.

Rehabilitation training after stroke is a long process, which is consistent with previous studies. $(25,29,56)$. The present study found that compared with 7 days, the volume of cerebral infarction and mNSS score decreased in 14 days. This change may be achieved through some protective responses, strong collateral circulation and favorable external intervention factors (26). These results suggest that treadmill exercise promotes the repair of brain injury by upregulating important molecules in the Wnt signaling pathway and increasing neurogenesis in the ischemic penumbra.

The current study used XAV-939, an inhibitor of the Wnt pathway, to determine the role of the Wnt pathway in the ischemic penumbra. When ischemic stroke occurs, the Wnt signaling pathway is activated. Inhibitors can aggravate neurological deficits and increase the volume of cerebral infarction. The present study found that on the 7 and 14th day after MCAO, the number of Nestin/BrdU- and Dcx/BrdU-positive cells in the IM group was decreased compared with the $\mathrm{M}$ group. This finding is consistent with the results of a previous study (19). Nissl staining and TEM results showed that neuronal damage was aggravated in the IM group compared with the $\mathrm{M}$ group. LFB staining showed a decrease in myelinated fibers in the IM group compared with the $\mathrm{M}$ group. The present results showed that inhibition of $\mathrm{Wnt} / \mathrm{\beta}$-catenin pathway can reduce neurogenesis and myelin repair in the ischemic penumbra.

In addition, inhibitors were used in the treadmill exercise group to explore the relationship between neuroprotection induced by treadmill exercise and $\mathrm{Wnt} / \beta$-catenin signaling pathway. At 7 and 14 days after MCAO, the number of Nestin/BrdU- and Dcx/BrdU-positive cells in the IEM group was decreased compared with the EM group, which was consistent with the low expression levels of Wnt3a, nuclear $\beta$-catenin and BDNF. LFB staining showed that compared with those in the EM group, the myelinated fibers in the IEM 

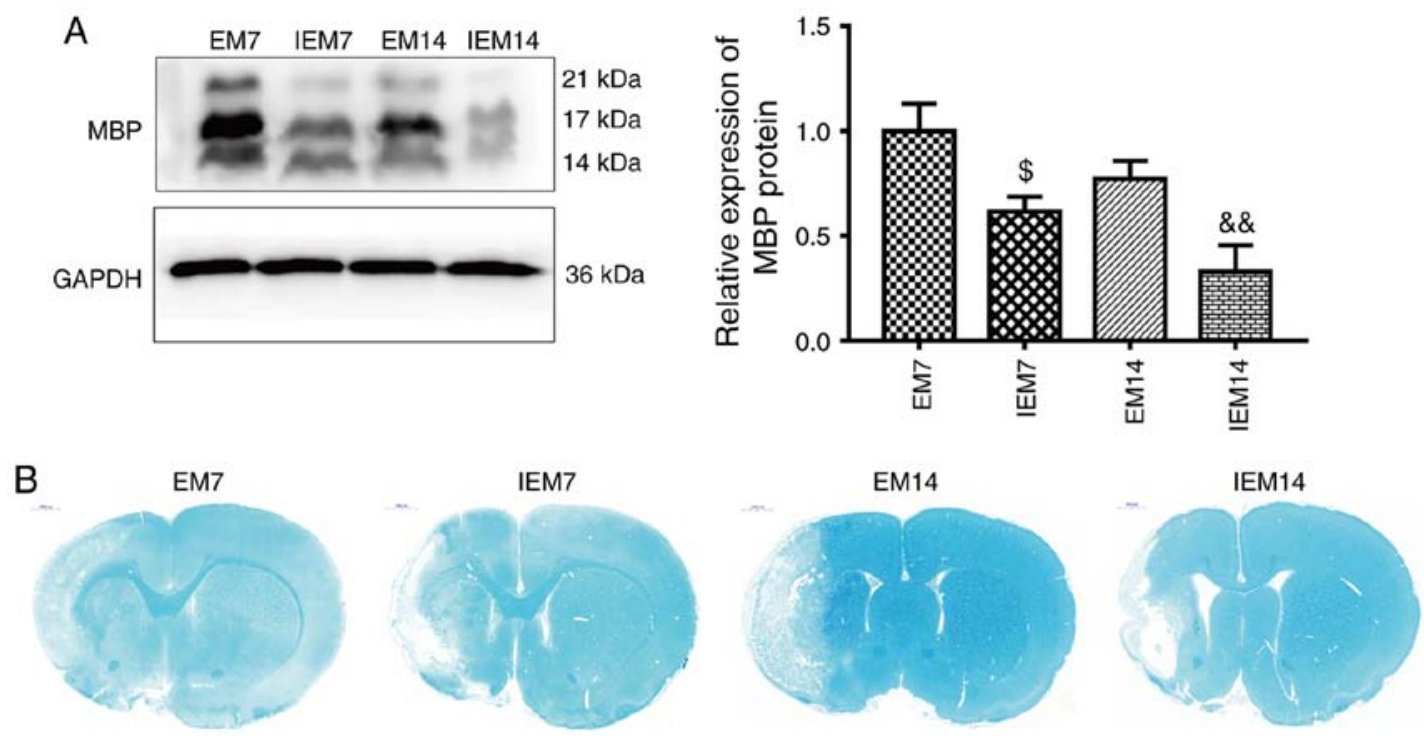

Figure 11. Inhibition of the Wnt/ $\beta$-catenin signaling pathway abolished exercise-promoted myelin repair in the ischemic penumbra. (A) Proteins expression levels and western blot analysis of MBP. Data are presented as the mean \pm standard deviation $(n=3)$. (B) Luxol fast blue staining in the ischemic penumbra after middle cerebral artery occlusion. ${ }^{\$} \mathrm{P}<0.05$ vs. the EM7 group; ${ }^{\& \&} \mathrm{P}<0.01$ vs. the EM14 group. MBP, myelin basic protein.

group decreased and the staining became lighter, which was consistent with the change in MBP expression. These results suggest that the inhibitor reverses the upregulation of Wnt3a, nuclear $\beta$-catenin and BDNF expression after treadmill training, reduces the expression of MBP, and weakens treadmill exercise-induced neurogenesis and myelin repair. It has been found that the neurovascular unit (NVU) is involved in brain injury and brain repair (26). Newly formed neuroblasts after stroke are located in the peri-infarction cortex and are closely related to the vascular endothelium (56). It has been found that the Wnt/ $\beta$-catenin signaling pathway can regulate vascular development and angiogenesis (57). Activation of the Wnt $/ \beta$-catenin signaling pathway after MCAO was reported to enhance the angiogenesis of the ischemic core (58). Wnt proteins are important mediators of intercellular communication. Wnt3a is involved in axonal regeneration after CNS injury. The latest studies on the CNS after injury in adults have shown that the classical Wnt signal transduction can induce axonal regeneration and neuronal growth, which indicates that the developmental effect of Wnt can be reused in adults through exogenous stimulation $(59,60)$. It has been found that promoting the expression of Wnt3a can increase vascular regeneration and vascular repair in the peri-infarct region (19). In the model of crush injury of the optic nerve in mice, intravitreal injection of classical Wnt/ $\beta$-catenin signal activator Wnt3a caused obvious axonal growth beyond the site of axonal lesion (61). Wnt3a signal transduction promotes neurite growth, increases neuronal function and induces repair after spinal cord contusion in adult rats (62). Previous studies have shown that exogenous Wnt3a mediated by lentivirus vectors enhances neurogenesis in vivo (20). In addition, exogenous Wnt3a can also promote the regeneration and differentiation of neural stem cells in vitro (63). In the present study, Wnt3a was focused on because of its established roles in neural development during embryogenesis, as well as in adult neurogenesis (64-66). Moreover, activation of Wnt3a/ $\beta$-catenin signaling directly regulates neurogenesis events, which includes a set of transcriptional changes leading to enhanced division and differentiation of $\mathrm{Dcx}^{+}$neuroblasts out of the SVZ $(12,67,68)$. Furthermore, Wnt3a is neuroprotective and may protect neurons during neurodegenerative processes, such as Alzheimer's disease and Huntington's disease (69-71). However, the relationship between neurogenesis and angiogenesis was not investigated in this study. Therefore, the current experiment can only prove that treadmill training promotes neurogenesis and myelin repair through the activation of the Wnt/ $\beta$-catenin signaling pathway. In contrast, inhibiting the conduction of the Wnt signaling pathway will weaken the neuroprotective effect induced by treadmill training. However, it is unclear whether angiogenesis or pairing of NVUs is directly or indirectly involved in neurogenesis and myelin repair produced by treadmill training through the activation of the $\mathrm{Wnt} / \beta$-catenin signaling pathway.

The present study has some limitations. First, the conditions to use real-time monitoring of cerebral blood flow unified modeling were not present, which may lead to modeling instability. Second, only a few signal pathway proteins were examined and no in vitro experiments were performed. Third, only neurogenesis in two periods after stroke (7 and 14 days) was studied; neurogenesis over a longer period of time, especially newborn mature neurons should be further studied. Fourth, except for LFB staining to observe the changes of myelin fibers and detect the expression level of the myelin marker protein MBP, no other analyses of proteins and cells related to myelin repair were conducted. In future experiments, the authors will work hard to improve the above deficiencies.

In conclusion, a new role for treadmill training in promoting neurogenesis and myelin sheath repair in juvenile rats was demonstrated. In addition, Wnt/ $\beta$-catenin signaling pathway was shown to not only mediate neurogenesis and myelin repair, but also participate in exercise-mediated neurogenesis and myelin repair. However, after ischemic stroke, treadmill-induced neurogenesis and myelin repair is a complex process, involving numerous key factors, which need to be fully clarified. 


\section{Acknowledgements}

Not applicable.

\section{Funding}

The present study was funded by a project funded by the Wenzhou Municipal Bureau of Science and Technology (grant nos. Y20170070 and Y20180096).

\section{Availability of data and materials}

The datasets used and/or analyzed during the current study are available from the corresponding author on reasonable request.

\section{Authors' contributions}

$\mathrm{XC}, \mathrm{HZ}$ and JC conceived and designed the experiment, and JC, QX and QH carried out the experiment. SW, JP, GP and YZ helped in the execution of the experiment; WS and LJ analyzed the data. JC and QX wrote the manuscript. All the authors discussed and suggested the experiment. All authors read and approved the final manuscript.

\section{Ethics approval and consent to participate}

All animal procedures in this study were approved by the Experimental Animal Ethics Committee of Wenzhou Medical University (ethical no. Wydw2019-0766) and carried out in accordance with the guidelines for the National Institutes of Health Guide for the Care and Use of Laboratory Animals.

\section{Patient consent for publication}

Not applicable.

\section{Competing interest}

The authors declare they have no competing interests.

\section{References}

1. Feigin VL, Norrving B, George MG, Foltz JL, Roth GA and Mensah GA: Prevention of stroke: A strategic global imperative. Nat Rev Neurol 12: 501-512, 2016.

2. Moskowitz MA, Lo EH and Iadecola C: The science of stroke: Mechanisms in search of treatments. Neuron 67: 181-198, 2010.

3. Ginsberg MD: Neuroprotection for ischemic stroke: Past, present and future. Neuropharmacology 55: 363-389, 2008.

4. Minnerup J, Sutherland BA, Buchan AM and Kleinschnitz C: Neuroprotection for stroke: Current status and future perspectives. Int J Mol Sci 13: 11753-11772, 2012.

5. Magnusson JP, Goritz C, Tatarishvili J, Dias DO, Smith EM, Lindvall $\mathrm{O}$, Kokaia $\mathrm{Z}$ and Frisén J: A latent neurogenic program in astrocytes regulated by Notch signaling in the mouse. Science 346: 237-241, 2014.

6. Ji Y, Teng L, Zhang R, Sun J and Guo Y: NRG-1 $\beta$ exerts neuroprotective effects against ischemia reperfusion-induced injury in rats through the JNK signaling pathway. Neuroscience 362: 13-24, 2017.

7. Astrup J, Siesjo BK and Symon L: Thresholds in cerebral ischemia-the ischemic penumbra. Stroke 12: 723-725, 1981.

8. Lo EH: A new penumbra: Transitioning from injury into repair after stroke. Nat Med 14: 497-500, 2008.

9. Jackman K and Iadecola C: Neurovascular regulation in the ischemic brain. Antioxid Redox Signal 22: 149-160, 2015.
10. Rodgers KM, Ahrendsen JT, Patsos OP, Strnad FA, Yonchek JC, Traystman RJ, Macklin WB and Herson PS: Endogenous neuronal replacement in the juvenile brain following cerebral ischemia. Neuroscience 380: 1-13, 2018.

11. Wang Y, Zhao Z, Rege SV, Wang M, Si G, Zhou Y, Wang S, Griffin JH, Goldman SA and Zlokovic BV: 3K3A-activated protein $\mathrm{C}$ stimulates postischemic neuronal repair by human neural stem cells in mice. Nat Med 22: 1050-1055, 2016.

12. Sun FL, Wang W, Zuo W, Xue JL, Xu JD, Ai HX, Zhang L, Wang XM and Ji XM: Promoting neurogenesis via Wnt/ $\beta$-catenin signaling pathway accounts for the neurorestorative effects of morroniside against cerebral ischemia injury. Eur J Pharmacol 738: 214-221, 2014.

13. Pak ME, Jung DH, Lee HJ, Shin MJ, Kim SY, Shin YB, Yun YJ, Shin HK and Choi BT: Combined therapy involving electroacupuncture and treadmill exercise attenuates demyelination in the corpus callosum by stimulating oligodendrogenesis in a rat model of neonatal hypoxia-ischemia. Exp Neurol 300: 222-231, 2018.

14. Hao XZ, Yin LK, Tian JQ, Li CC, Feng XY, Yao ZW, Jiang M and Yang YM: Inhibition of Notch1 signaling at the subacute stage of stroke promotes endogenous neurogenesis and motor recovery after stroke. Front Cell Neurosci 12: 245, 2018.

15. Danzer SC: Postnatal and adult neurogenesis in the development of human disease. Neuroscientist 14: 446-458, 2008.

16. Piccin D and Morshead CM: Wnt signaling regulates symmetry of division of neural stem cells in the adult brain and in response to injury. Stem Cells 29: 528-538, 2011.

17. Yoshinaga Y, Kagawa T, Shimizu T, Inoue T, Takada S, Kuratsu J and Taga T: Wnt3a promotes hippocampal neurogenesis by shortening cell cycle duration of neural progenitor cells. Cell Mol Neurobiol 30: 1049-1058, 2010.

18. Munji RN, Choe Y, Li G, Siegenthaler JA and Pleasure SJ: Wnt signaling regulates neuronal differentiation of cortical intermediate progenitors. J Neurosci 31: 1676-1687, 2011.

19. Wei ZZ, Zhang JY, Taylor TM, Gu X, Zhao Y and Wei L: Neuroprotective and regenerative roles of intranasal Wnt-3a administration after focal ischemic stroke in mice. J Cereb Blood Flow Metab 38: 404-421, 2018

20. Lie DC, Colamarino SA, Song HJ, Désiré L, Mira H, Consiglio A, Lein ES, Jessberger S, Lansford H, Dearie AR and Gage FH: Wnt signalling regulates adult hippocampal neurogenesis. Nature 437: 1370-1375, 2005

21. Qu Q, Sun G, Murai K, Ye P, Li W, Asuelime G, Cheung YT and Shi Y: Wnt7a regulates multiple steps of neurogenesis. Mol Cell Biol 33: 2551-2559, 2013.

22. Wang J, Chen T and Shan G: MiR-148b regulates proliferation and differentiation of neural stem cells via Wnt $/ \beta$-catenin signaling in rat ischemic stroke model. Front Cell Neurosci 11: $329,2017$.

23. Xu D, Hou K, Li F, Chen S, Fang W and Li Y: XQ-1H alleviates cerebral ischemia in mice through inhibition of apoptosis and promotion of neurogenesis in a $\mathrm{Wnt} / \beta$-catenin signaling dependent way. Life Sci 235: 116844, 2019.

24. Adachi K, Mirzadeh Z, Sakaguchi M, Yamashita T, Nikolcheva T, Gotoh Y, Peltz G, Gong L, Kawase T, Alvarez-Buylla A, et al: Beta-catenin signaling promotes proliferation of progenitor cells in the adult mouse subventricular zone. Stem Cells 25: 2827-2836, 2007.

25. Xie Q, Cheng J, Pan G, Wu S, Hu Q, Jiang H, Wang Y, Xiong J, Pang Q and Chen X: Treadmill exercise ameliorates focal cerebral ischemia/reperfusion-induced neurological deficit by promoting dendritic modification and synaptic plasticity via upregulating caveolin-1/VEGF signaling pathways. Exp Neurol 313: 60-78, 2019.

26. Chen Z, Hu Q, Xie Q, Wu S, Pang Q, Liu M, Zhao Y, Tu F, Liu C and Chen X: Effects of treadmill exercise on motor and cognitive function recovery of MCAO mice through the caveolin-1/VEGF signaling pathway in ischemic penumbra. Neurochem Res 44: 930-946, 2019.

27. Schabitz WR, Berger C, Kollmar R, Seitz M, Tanay E, Kiessling M, Schwab S and Sommer C: Effect of brain-derived neurotrophic factor treatment and forced arm use on functional motor recovery after small cortical ischemia. Stroke 35: 992-997, 2004.

28. Ahn JH, Choi JH, Park JH, Kim IH, Cho JH, Lee JC, Koo HM, Hwangbo G, Yoo KY, Lee CH, et al: Long-term exercise improves memory deficits via restoration of myelin and microvessel damage, and enhancement of neurogenesis in the aged gerbil hippocampus after ischemic stroke. Neurorehabil Neural Repair 30: 894-905, 2016. 
29. Pang Q, Zhang H, Chen Z, Wu Y, Bai M, Liu Y, Zhao Y, Tu F, Liu $C$ and Chen X: Role of caveolin-1/vascular endothelial growth factor pathway in basic fibroblast growth factor-induced angiogenesis and neurogenesis after treadmill training following focal cerebral ischemia in rats. Brain Res 1663: 9-19, 2017.

30. Zhao Y, Pang Q, Liu M, Pan J, Xiang B, Huang T, Tu F, Liu C and Chen X: Treadmill exercise promotes neurogenesis in ischemic rat brains via caveolin-1/VEGF signaling pathways. Neurochem. Res 42: 389-397, 2017.

31. Saver JL, Albers GW, Dunn B, Johnston KC and Fisher M; STAIR VI Consortium: Stroke Therapy Academic Industry Roundtable (STAIR) recommendations for extended window acute stroke therapy trials. Stroke 40: 2594-2600, 2009

32. Longa EZ, Weinstein PR, Carlson S and Cummins R: Reversible middle cerebral artery occlusion without craniectomy in rats Stroke 20: 84-91,1989.

33. Jean LeBlanc N, Menet R, Picard K, Parent G, Tremblay ME and ElAli A: Canonical Wnt pathway maintains blood-brain barrier integrity upon ischemic stroke and its activation ameliorates tissue plasminogen activator therapy. Mol Neurobiol 56: 6521-6538, 2019

34. Wang YZ, Yamagami T, Gan Q, Wang Y, Zhao T, Hamad S, Lott P, Schnittke N, Schwob JE and Zhou CJ: Canonical Wnt signaling promotes the proliferation and neurogenesis of peripheral olfactory stem cells during postnatal development and adult regeneration. J Cell Sci 124: 1553-1563, 2011.

35. Chen J, Li Y, Wang L, Zhang Z, Lu D, Lu M and Chopp M: Therapeutic benefit of intravenous administration of bone marrow stromal cells after cerebral ischemia in rats. Stroke 32 1005-1011, 2001.

36. Ding YH, Ding Y, Li J, Bessert DA and Rafols JA: Exercise pre-conditioning strengthens brain microvascular integrity in a rat stroke model. Neurol Res 28: 184-189, 2006.

37. Gao Y, Zhao Y, Pan J, Yang L, Huang T, Feng X, Li C, Liang S, Zhou D, Liu C, et al: Treadmill exercise promotes angiogenesis in the ischemic penumbra of rat brains through caveolin-1/VEGF signaling pathways. Brain Res 1585: 83-90, 2014.

38. Ashwal S, Tone B, Tian HR, Cole DJ, Liwnicz BH and Pearce WJ: Core and penumbral nitric oxide synthase activity during cerebral ischemia and reperfusion in the rat pup. Pediatr Res 46: 390-400, 1999.

39. Zhao C, Deng W and Gage FH: Mechanisms and functional implications of adult neurogenesis. Cell 132: 645-660, 2008

40. Wang X, Zhang M, Yang SD, Li WB, Ren SQ, Zhang J and Zhang F: Pre-ischemic treadmill training alleviates brain damage via GLT-1-mediated signal pathway after ischemic stroke in rats. Neuroscience 274: 393-402, 2014

41. Kim HN, Pak ME, Shin MJ, Kim SY, Shin YB, Yun YJ, Shin HK and Choi BT: Comparative analysis of the beneficial effects of treadmill training and electroacupuncture in a rat model of neonatal hypoxia-ischemia. Int J Mol Med 39: 1393-1402, 2017.

42. Gallo V and Armstrong RC: Myelin repair strategies: A cellular view. Curr Opin Neurol 21: 278-283, 2008

43. Dewar D, Underhill SM and Goldberg MP: Oligodendrocytes and ischemic brain injury. J Cereb Blood Flow Metab 23: 263-274, 2003.

44. Micu I, Jiang Q, Coderre E, Ridsdale A, Zhang L, Woulfe J, Yin X, Trapp BD, McRory JE, Rehak R, et al: NMDA receptors mediate calcium accumulation in myelin during chemical ischaemia. Nature 439: 988-992, 2006.

45. McTigue DM and Tripathi RB: The life, death, and replacement of oligodendrocytes in the adult CNS. J Neurochem 107: 1-19, 2008.

46. Yi H, Hu J, Qian J and Hackam AS: Expression of brain-derived neurotrophic factor is regulated by the Wnt signaling pathway. Neuroreport 23: 189-194, 2012

47. Lu B, Nagappan G, Guan X, Nathan PJ and Wren P: BDNF-based synaptic repair as a disease-modifying strategy for neurodegenerative diseases. Nat Rev Neurosci 14: 401-416, 2013.

48. Bekinschtein P, Oomen CA, Saksida LM and Bussey TJ: Effects of environmental enrichment and voluntary exercise on neurogenesis, learning and memory, and pattern separation: BDNF as a critical variable? Semin Cell Dev Biol 22: 536-542, 2011.

49. Greenberg ME, Xu B, Lu B and Hempstead BL: New insights in the biology of BDNF synthesis and release: Implications in CNS function. J Neurosci 29: 12764-12767, 2009.

50. Brigadski T, Hartmann M and Lessmann V: Differential vesicular targeting and time course of synaptic secretion of the mammalian neurotrophins. J Neurosci 25: 7601-7614, 2005.

51. Matsuda N, Lu H, Fukata Y, Noritake J, Gao H, Mukherjee S, Nemoto T,Fukata M and Poo MM: Differential activity-dependent secretion of brain-derived neurotrophic factor from axon and dendrite. J Neurosci 29: 14185-14198, 2009.
52. Scharfman H, Goodman J, Macleod A, Phani S, Antonelli C and Croll S: Increased neurogenesis and the ectopic granule cells after intrahippocampal BDNF infusion in adult rats. Exp Neurol 192: 348-356, 2005

53. Benraiss A, Chmielnicki E, Lerner K, Roh D and Goldman SA: Adenoviral brain-derived neurotrophic factor induces both neostriatal and olfactory neuronal recruitment from endogenous progenitor cells in the adult forebrain. J Neurosci 21: 6718-6731, 2001.

54. Jones KR, Farinas I, Backus C and Reichardt LF: Targeted disruption of the BDNF gene perturbs brain and sensory neuron development but not motor neuron development. Cell 76: 989-999, 1994

55. Obernier K, Tong CK and Alvarez-Buylla A: Restricted nature of adult neural stem cells: Re-evaluation of their potential for brain repair. Front Neurosci 8: 162, 2014.

56. Carmichael ST, Ohab J and Nguyen J: Post-stroke neurogenesis and the neurovascular niche: Newly born neuroblasts localize to peri-infarct cortex in close association with the vascular endothelium. J Cereb Blood Flow Metab 25: S214-S214, 2005.

57. Parmalee NL and Kitajewski J: Wnt signaling in angiogenesis. Curr Drug Targets 9: 558-564, 2008

58. Xu Y, Zhang G, Kang Z, Xu Y, Jiang W and Zhang S: Cornin increases angiogenesis and improves functional recovery after stroke via the Ang1/Tie 2 axis and the Wnt/ $\beta$-catenin pathway. Arch Pharm Res 39: 133-142, 2016.

59. Ciani $\mathrm{L}$ and Salinas PC: WNTs in the vertebrate nervous system: From patterning to neuronal connectivity. Nat Rev Neurosci 6: 351-362, 2005

60. Salinas PC: Wnt signaling in the vertebrate central nervous system: From axon guidance to synaptic function. Cold Spring Harb Perspect Biol 4: pii: a008003, 2012.

61. Patel AK, Park KK and Hackam AS: Wnt signaling promotes axonal regeneration following optic nerve injury in the mouse. Neuroscience 343: 372-383, 2017.

62. Yin ZS, Zu B, Chang J and Zhang H: Repair effect of Wnt3a protein on the contused adult rat spinal cord. Neurol Res 30: 480-486, 2008

63. Kalani MY, Cheshier SH, Cord BJ, Bababeygy SR, Vogel H, Weissman IL, Palmer TD and Nusse R: Wnt-mediated self-renewal of neural stem/progenitor cells. Proc Natl Acad Sci USA 105: 16970-16975, 2008.

64. Ikeya M and Takada S: Wnt-3a is required for somite specification along the anteroposterior axis of the mouse embryo and for regulation of cdx-1 expression. Mech Dev 103: 27-33, 2001.

65. Mastroiacovo F, Busceti CL, Biagioni F, Moyanova SG, Meisler MH, Battaglia G, Caricasole A, Bruno V and Nicoletti F: Induction of the Wnt antagonist, Dickkopf-1, contributes to the development of neuronal death in models of brain focal ischemia. J Cereb Blood Flow Metab 29: 264-276, 2009.

66. Mussmann C, Hubner R, Trilck M, Rolfs A and Frech MJ: HES5 is a key mediator of Wnt-3a-induced neuronal differentiation. Stem Cells Dev 23: 1328-1339, 2014.

67. Giuliani D, Zaffe D, Ottani A, Spaccapelo L, Galantucci M, Minutoli L, Bitto A, Irrera N, Contri M, Altavilla D, et al: Treatment of cerebral ischemia with melanocortins acting at MC4 receptors induces marked neurogenesis and long-lasting functional recovery. Acta Neuropathol 122: 443-453, 2011.

68. Spaccapelo L, Galantucci M, Neri L, Contri M, Pizzala R, D'Amico R, Ottani A, Sandrini M, Zaffe D, Giuliani D and Guarini S: Up-regulation of the canonical Wnt-3A and Sonic hedgehog signaling underlies melanocortin-induced neurogenesis after cerebral ischemia. Eur J Pharmacol 707: 78-86, 2013.

69. Inestrosa NC, Urra S and Colombres M: Acetylcholinesterase (AChE)-amyloid-beta-peptide complexes in Alzheimer's disease. The Wnt signaling pathway. Curr Alzheimer Res 1: 249-254, 2004.

70. Toledo EM, Colombres M and Inestrosa NC: Wnt signaling in neuroprotection and stem cell differentiation. Prog Neurobiol 86: 281-296, 2008

71. Tourette C, Farina F, Vazquez-Manrique RP, Orfila AM, Voisin J, Hernandez S, Offner N, Parker JA, Menet S, Kim J, et al: The Wnt receptor Ryk reduces neuronal and cell survival capacity by repressing FOXO activity during the early phases of mutant huntingtin pathogenicity. PLoS Biol 12: e1001895, 2014.

This work is licensed under a Creative Commons Attribution-NonCommercial-NoDerivatives 4.0 International (CC BY-NC-ND 4.0) License. 